PAPER

\section{Fluctuations in the uniform shear flow state of a granular gas}

To cite this article: M I García de Soria et al J. Stat. Mech. (2014) P09024

View the article online for updates and enhancements.

\section{Related content}

- $\frac{\text { Hydrodynamic modes, Green-Kubo }}{\text { relations, and velocity correlations in dilute }}$
$\frac{\text { granulargases }}{\text { J Javier Brey, M J Ruiz-Montero, P }}$
Maynar et al.
- Shear-rate-dependent transport
$\frac{\text { coefficients for inelastic Maxwell models }}{\text { Vicente Garzó }}$
- Lattice models for granular-like velocity
fields: finite-size effects
C A Plata, A Manacorda, A Lasanta et al.

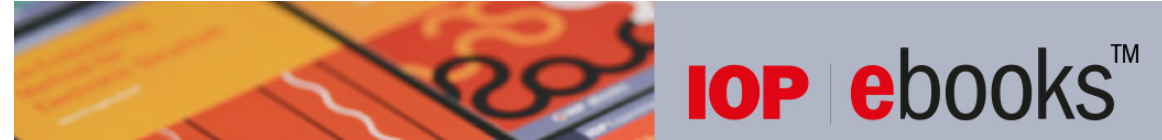

Bringing together innovative digital publishing with leading authors from the global scientific community. Start exploring the collection-download the first chapter of every title for free. 


\title{
Fluctuations in the uniform shear flow state of a granular gas
}

\section{I García de Soria, P Maynar and J Javier Brey}

Física Teórica, Universidad de Sevilla, Apartado de Correos 1065, E-41080, Sevilla, Spain

E-mail: maynar@us.es

Received 30 June 2014

Accepted for publication 17 July 2014

Published 22 September 2014

Online at stacks.iop.org/JSTAT/2014/P09024

doi:10.1088/1742-5468/2014/09/P09024

\begin{abstract}
Using kinetic theory we analyse the fluctuations of the total internal energy of a granular gas under stationary uniform shear flow, and find that they are coupled to fluctuations in the different components of the total pressure tensor. Explicit expressions for all the possible one- and two-time cross and auto correlations of the fluctuations are obtained for the two dimensional case. For the range of inelasticity considered, good agreement is found between the theoretical predictions and Molecular Dynamics simulations.
\end{abstract}

Keywords: granular matter, fluctuations (theory), Boltzmann equation 


\section{Contents}

1. Introduction 2

2. Kinetic equations for the model 4

3. The stationary uniform shear flow state $\quad 6$

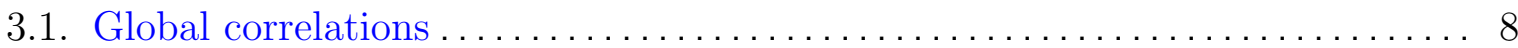

3.2. Correlations between global quantities ....................... 10

4. Fluctuations of the total internal energy $\quad 10$

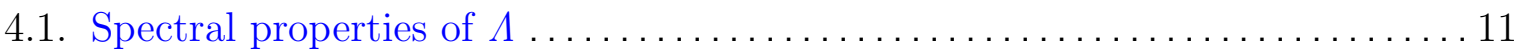

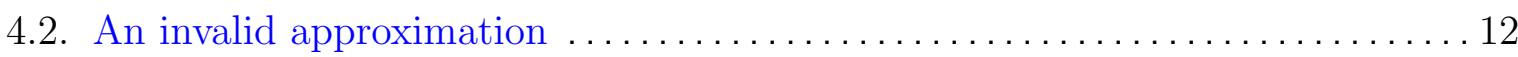

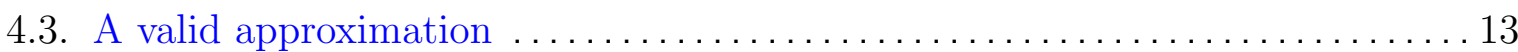

5. Fluctuations of the relevant global quantities $\quad 17$

6. Simulation results 20

7. Conclusion and discussion 24

$\begin{array}{ll}\text { Acknowledgments } & \mathbf{2 5}\end{array}$

Appendix A. Evolution equation for the temperature 25

Appendix B. Validity of the approximation given by equation (111) 26

$\begin{array}{ll}\text { Appendix C. Evaluation of the } C_{i j} \text { coefficients } & 27\end{array}$

$\begin{array}{ll}\text { References } & 31\end{array}$

\section{Introduction}

A granular system can be defined as an ensemble of macroscopic particles (grains) that collide inelastically, i.e. kinetic energy is dissipated in collisions. When the dynamics of the grains can be partitioned into sequences of two-body collisions, the system is referred to as a granular gas, and the reliability of a kinetic theory description is supported both by experiments and computer simulations [1-5]. One of the models most frequently used to study granular gases is the Inelastic Hard Sphere (IHS) model, the dynamics of which comprise free streaming followed by instantaneous inelastic collisions. For this model, all the techniques of kinetic theory can be applied [6]. In particular, in the low density limit, the dynamics of the one-particle distribution function are given by the inelastic Boltzmann equation $[7,8]$, and the correlation functions obey a closed set of equations [9].

Macroscopically it is known that, in many cases, the dynamics of a granular system resemble those of a fluid. For dilute systems, hydrodynamic equations can be derived 
by applying the Chapman-Enskog expansion [10] or linear response methods [1113], obtaining explicit expressions for the transport coefficients. In all these studies, the Homogeneous Cooling State (HCS) plays a particularly important role. This is a homogeneous state in which all the time dependence in the one-particle distribution function goes through the granular temperature (defined as the second velocity moment of the velocity distribution). Due to the inelasticity of collisions, the temperature decays monotonically in time [14]. It is known that, for a wide range of initial conditions, the HCS is reached in the long time limit for isolated granular gases. Thus this state plays, for granular gases, a role similar to the equilibrium state for molecular, elastic fluids. In fact, the zeroth order of the gradient's distribution in the Chapman-Enskog expansion is a 'local' HCS [10].

Despite this analogy with normal fluids, there are important differences. Due to the macroscopic character of the grains, a granular system typically contains far fewer particles than a normal fluid. The macroscopic field fluctuations are therefore of special relevance not only theoretically, but also from a practical point of view. Total energy fluctuations have been studied in the HCS, and explicit expressions for its variance and two-time correlation function have been obtained [9]. With some generality, Langevin-like equations for the fluctuating hydrodynamic fields have been derived using the NavierStokes equations $[15,16]$. Fluctuation-dissipation theorems of the second kind were not present, i.e. the amplitude of the noise is not related to the transport coefficients. However, the two-time correlation functions decay as a macroscopic perturbation so that fluctuation-dissipation theorems of first kind hold [17].

The study of fluctuations in the HCS is of special relevance, because it serves as a starting point for generalization to other states. By analogy with normal fluids, the equations for the fluctuating fields can be written intuitively for states that are close to the HCS. The deterministic part of the equations is the linearization of the macroscopic equations around the particular state considered. The noise can be assumed to have the same stochastic properties as that in the HCS but with the total fields replaced by the local actual ones. This should be valid if the state is not far from the HCS, which is the case for small gradients. This idea has been applied in [18] to calculate the total internal energy fluctuations in the stationary Uniform Shear Flow (USF) state. This state has a uniform density, a constant and uniform temperature, and a flow velocity with a linear profile. Due to its simplicity, it has been extensively studied [19-24]. The theoretical predictions of [18] were expected to hold only for small gradients. For the stationary USF state that means small inelasticity, due to the coupling between gradients and inelasticity, which is a characteristic feature of stationary states of granular systems. This work examines fluctuations of the total internal energy in the stationary USF state using kinetic theory tools. This permits analysis of the problem in general, without any limitation to small inelasticity. In particular, differences from the "local" HCS results of [18] will be discussed, extending these results to finite dissipation. It will be shown that the structure of these fluctuations is more complex than expected, since they are coupled to the fluctuations of the several components of the total pressure tensor. Finally, explicit results will be obtained through a systematic and controlled expansion in the degree of inelasticity.

The plan of the paper is as follows. In the next section, the IHS model is described in some detail, and the evolution equations for the relevant distributions are summarized. These equations are applied to the stationary USF state in section 3, where correlations of 
global quantities are specifically considered. In section 4 we study fluctuations of the total internal energy and show that they are coupled to the components of the total pressure tensor. Section 5 is a complete study of all the fluctuations. In section 6 the analytical predictions are compared with Molecular Dynamics simulations, finding, in general, a good agreement. The final section contains some general conclusions and comments.

\section{Kinetic equations for the model}

The system we consider is a dilute gas of $N$ smooth inelastic hard spheres $(d=3)$ or disks $(d=2)$ of mass $m$ and diameter $\sigma$. Let $X_{i}(t) \equiv\left\{\mathbf{R}_{i}(t), \mathbf{V}_{i}(t)\right\}$ denote the position and velocity of particle $i$ at time $t$. The dynamic state of the system, $\Gamma(t) \equiv\left\{X_{1}(t), \ldots, X_{N}(t)\right\}$, is generated by free streaming followed by instantaneous inelastic collisions characterized by a coefficient of normal restitution, $\alpha$, independent of the relative velocity. If at time $t$ there is a binary encounter between particles $i$ and $j$, with velocities $\mathbf{V}_{i}(t)$ and $\mathbf{V}_{j}(t)$ respectively, the postcollisional velocities $\mathbf{V}_{i}^{\prime}(t)$ and $\mathbf{V}_{j}^{\prime}(t)$ are

$$
\begin{aligned}
& \mathbf{V}_{i}^{\prime}=\mathbf{V}_{i}-\frac{1+\alpha}{2}\left(\hat{\boldsymbol{\sigma}} \cdot \mathbf{V}_{i j}\right) \hat{\boldsymbol{\sigma}}, \\
& \mathbf{V}_{j}^{\prime}=\mathbf{V}_{j}+\frac{1+\alpha}{2}\left(\hat{\boldsymbol{\sigma}} \cdot \mathbf{V}_{i j}\right) \hat{\boldsymbol{\sigma}},
\end{aligned}
$$

where $\mathbf{V}_{i j} \equiv \mathbf{V}_{i}-\mathbf{V}_{j}$ is the relative velocity and $\hat{\boldsymbol{\sigma}}$ is the unit vector pointing from the center of particle $j$ to the center of particle $i$ at contact.

Microscopic densities in phase space, $F_{s}\left(x_{1}, \ldots, x_{s}, t\right)$, are defined by

$$
\begin{aligned}
& F_{1}\left(x_{1}, t\right)=\sum_{i=1}^{N} \delta\left[x_{1}-X_{i}(t)\right], \\
& F_{2}\left(x_{1}, x_{2}, t\right)=\sum_{i=1}^{N} \sum_{j \neq i}^{N} \delta\left[x_{1}-X_{i}(t)\right] \delta\left[x_{2}-X_{j}(t)\right],
\end{aligned}
$$

etc, where we have introduced the field variables, $x_{i} \equiv\left\{\mathbf{r}_{i}, \mathbf{v}_{i}\right\}$. The averages of the microscopic densities over the probability distribution function, $\rho(\Gamma, 0)$, characterizing the initial state, are the usual one-time reduced distribution functions

$$
f_{s}\left(x_{1}, \ldots, x_{s}, t\right) \equiv\left\langle F_{s}\left(x_{1}, \ldots, x_{s}, t\right)\right\rangle,
$$

where we have introduced the notation

$$
\langle G\rangle \equiv \int \mathrm{d} \Gamma G(\Gamma) \rho(\Gamma, 0) .
$$

Two-time reduced distribution functions can also be defined in terms of the microscopic densities as

$$
f_{r, s}\left(x_{1}, \ldots, x_{r}, t ; x_{1}, \ldots, x_{s}, t^{\prime}\right) \equiv\left\langle F_{r}\left(x_{1}, \ldots, x_{r}, t\right) F_{s}\left(x_{1}, \ldots, x_{s}, t^{\prime}\right)\right\rangle,
$$

where it will be assumed that $t>t^{\prime}>0$. Evolution equations for the reduced distributions can be derived from first principles [8,9], as for the elastic case [25]. The one-time reduced distribution functions obey the generalization for inelastic collisions of the Bogoliubov, Born, Green, Kirkwood and Yvon hierarchy, but its general application is limited because 
the equations are not closed. The same applies to the two-time reduced distribution functions.

It is convenient to introduce correlation functions through the usual cluster expansion. From the one-time reduced distributions, one-time correlations, $g_{s}\left(x_{1}, \ldots, x_{s}, t\right)$, are defined by

$$
\begin{gathered}
f_{2}\left(x_{1}, x_{2}, t\right) \equiv f_{1}\left(x_{1}, t\right) f_{1}\left(x_{2}, t\right)+g_{2}\left(x_{1}, x_{2}, t\right), \\
f_{3}\left(x_{1}, x_{2}, x_{3}, t\right) \equiv f_{1}\left(x_{1}, t\right) f_{1}\left(x_{2}, t\right) f_{1}\left(x_{3}, t\right)+f_{1}\left(x_{1}, t\right) g_{2}\left(x_{2}, x_{3}, t\right) \\
+f_{1}\left(x_{2}, t\right) g_{2}\left(x_{1}, x_{3}, t\right)+f_{1}\left(x_{3}, t\right) g_{2}\left(x_{1}, x_{2}, t\right)+g_{3}\left(x_{1}, x_{2}, x_{3}, t\right),
\end{gathered}
$$

etc. Similarly, two-time correlation functions, $h_{r, s}\left(x_{1}, \ldots, x_{r}, t ; x_{1}, \ldots, x_{s}, t^{\prime}\right)$, can be defined. In particular, $h_{1,1}$ and $h_{2,1}$ are introduced through

$$
\begin{aligned}
& f_{1,1}\left(x_{1}, t ; x_{1}^{\prime}, t^{\prime}\right)=f_{1}\left(x_{1}, t\right) f_{1}\left(x_{1}^{\prime}, t^{\prime}\right)+h_{1,1}\left(x_{1}, t ; x_{1}^{\prime}, t^{\prime}\right), \\
& f_{2,1}\left(x_{1}, x_{2}, t ; x_{1}^{\prime}, t^{\prime}\right)=f_{1}\left(x_{1}, t\right) f_{1}\left(x_{2}, t\right) f_{1}\left(x_{1}^{\prime}, t^{\prime}\right)+g_{2}\left(x_{1}, x_{2}, t\right) f_{1}\left(x_{1}^{\prime}, t\right) \\
& +h_{1,1}\left(x_{1}, t ; x_{1}^{\prime}, t^{\prime}\right) f_{1}\left(x_{2}, t\right)+h_{1,1}\left(x_{2}, t ; x_{1}^{\prime}, t^{\prime}\right) f_{1}\left(x_{1}, t\right) \\
& +h_{2,1}\left(x_{1}, x_{2}, t ; x_{1}^{\prime}, t^{\prime}\right) .
\end{aligned}
$$

In the low density limit and for distances much larger than the diameter of the particles, a closed set of equations for $f_{1}, g_{2}$ and $h_{1,1}$ is obtained $[9,25]$.

The one-particle distribution function satisfies the inelastic Boltzmann equation $[7,8]$

$$
\left[\frac{\partial}{\partial t}+L^{(0)}\left(x_{1}\right)\right] f_{1}\left(x_{1}, t\right)=J\left[x_{1}, t \mid f_{1}\right]
$$

where we have introduced the free-streaming operator

$$
L^{(0)}\left(x_{1}\right)=\mathbf{v}_{1} \cdot \frac{\partial}{\partial \mathbf{r}_{1}} .
$$

The collisional term reads

$$
J\left[x_{1}, t \mid f_{1}\right]=\int \mathrm{d} x_{2} \delta\left(\mathbf{r}_{12}\right) \bar{T}_{0}\left(\mathbf{v}_{1}, \mathbf{v}_{2}\right) f_{1}\left(x_{1}, t\right) f_{1}\left(x_{2}, t\right),
$$

with the binary collision operator, $T_{0}$, given by

$$
\bar{T}_{0}\left(\mathbf{v}_{1}, \mathbf{v}_{2}\right)=\sigma^{d-1} \int \mathrm{d} \hat{\boldsymbol{\sigma}} \Theta\left(\mathbf{v}_{12} \cdot \hat{\boldsymbol{\sigma}}\right)\left(\mathbf{v}_{12} \cdot \hat{\boldsymbol{\sigma}}\right)\left[\alpha^{-2} b_{\boldsymbol{\sigma}}^{-1}(1,2)-1\right] .
$$

Here $\boldsymbol{\sigma}=\sigma \hat{\boldsymbol{\sigma}}, \mathrm{d} \hat{\boldsymbol{\sigma}}$ is the solid angle element for $\hat{\boldsymbol{\sigma}}, \mathbf{v}_{12} \equiv \mathbf{v}_{1}-\mathbf{v}_{2}, \Theta$ is the Heaviside step function and the operator $b_{\boldsymbol{\sigma}}^{-1}(1,2)$ replaces all the velocities $\mathbf{v}_{1}$ and $\mathbf{v}_{2}$ appearing to its right by the precollisional values $\mathbf{v}_{1}^{*}$ and $\mathbf{v}_{2}^{*}$,

$$
\begin{aligned}
& \mathbf{v}_{1}^{*} \equiv b_{\boldsymbol{\sigma}}^{-1}(1,2) \mathbf{v}_{1}=\mathbf{v}_{1}-\frac{1+\alpha}{2 \alpha}\left(\hat{\boldsymbol{\sigma}} \cdot \mathbf{v}_{12}\right) \hat{\boldsymbol{\sigma}}, \\
& \mathbf{v}_{2}^{*} \equiv b_{\boldsymbol{\sigma}}^{-1}(1,2) \mathbf{v}_{2}=\mathbf{v}_{2}+\frac{1+\alpha}{2 \alpha}\left(\hat{\boldsymbol{\sigma}} \cdot \mathbf{v}_{12}\right) \hat{\boldsymbol{\sigma}} .
\end{aligned}
$$

The equation for the one-time correlation function in the low density limit is

$$
\begin{gathered}
{\left[\frac{\partial}{\partial t}+L^{(0)}\left(x_{1}\right)+L^{(0)}\left(x_{2}\right)-K\left[x_{1}, t \mid f_{1}\right]-K\left[x_{2}, t \mid f_{1}\right]\right] g_{2}\left(x_{1}, x_{2}, t\right)} \\
=\delta\left(\mathbf{r}_{12}\right) \bar{T}_{0}\left(\mathbf{v}_{1}, \mathbf{v}_{2}\right) f_{1}\left(x_{1}, t\right) f_{1}\left(x_{2}, t\right),
\end{gathered}
$$


where we have introduced the linear operator

$$
K\left[x_{i}, t \mid f_{1}\right] \equiv \int \mathrm{d} x_{3} \delta\left(\mathbf{r}_{i 3}\right) \bar{T}_{0}\left(\mathbf{v}_{i}, \mathbf{v}_{3}\right)\left(1+P_{i 3}\right) f_{1}\left(x_{3}, t\right) .
$$

The operator $P_{i j}$ interchanges the labels of particle $i$ and $j$ in the quantities to its right. Basically, equation (16) shows that velocity correlations between particles with velocities $\mathbf{v}_{1}$ and $\mathbf{v}_{2}$ are generated by uncorrelated collisions, implying particles with velocities $\mathbf{v}_{1}$ and $\mathbf{v}_{2}$ through the right hand side of equation (16), and correlated collisions implying two particles with velocities $\mathbf{v}_{1}$ or $\mathbf{v}_{2}$ and a third particle with velocity $\mathbf{v}_{3}$ through the linear operator $K$.

Finally, consider the two-time correlations. In the same limit, the following equation for the first of these is obtained

$$
\left[\frac{\partial}{\partial t}+L^{(0)}\left(x_{1}\right)-K\left[x_{1}, t \mid f_{1}\right]\right] h_{1,1}\left(x_{1}, t ; x_{1}^{\prime}, t^{\prime}\right)=0
$$

This equation has to be solved with the initial condition

$$
h_{1,1}\left(x_{1}, t^{\prime} ; x_{1}^{\prime}, t^{\prime}\right)=f_{1}\left(x_{1}, t^{\prime}\right) \delta\left(x_{1}-x_{1}^{\prime}\right)+g_{2}\left(x_{1}, x_{1}^{\prime}, t^{\prime}\right),
$$

that follows directly from the definitions in equations (6) and (9). If we consider states with a one-particle distribution function, $\tilde{f}_{1}$, very close to a given reference distribution, $f_{1}$, the difference of both distributions, $\delta f_{1} \equiv \tilde{f}_{1}-f_{1}$, fulfils to linear order

$$
\left[\frac{\partial}{\partial t}+L^{(0)}\left(x_{1}\right)-K\left[x_{1}, t \mid f_{1}\right]\right] \delta f_{1}\left(x_{1}, t\right)=0
$$

where $K$ is given by equation (17). The structure of these equations is important because it shows that the two-time correlations in a given state decay in the same way that a linear perturbation of the one-particle distribution function around this state decays. This is because the linear operator governing the dynamics is, in both cases, the operator $K$ given by equation (17). Of course, although the initial condition of equation (20) is free (the only restriction being $\left|\delta f_{1}\left(x_{1}, t^{\prime}\right)\right| \ll f_{1}\left(x_{1}, t^{\prime}\right)$ ), the initial condition for equation (18) is given by equation (19).

\section{The stationary uniform shear flow state}

In this section we will apply the equations of the previous section to the stationary USF state. At a macroscopic level, this state is characterized by a uniform number density, $n_{s}$, a stationary temperature, $T_{s}$, and a constant velocity field with a linear profile, $\mathbf{u}_{s}=a y \hat{\mathbf{e}}_{x}$, where $a$ is the constant shear rate and $\hat{\mathbf{e}}_{x}$ is a unit vector in the direction of the $x$-axis (the subindex $s$ has been introduced to label the state) [20-23]. In this stationary state, cooling due to collisions is offset by viscous heating

$$
\frac{2 a}{\mathrm{~d} n_{s}} P_{x y, s}=\zeta_{s} T_{s}
$$

where $P_{x y, s}$ is the $x y$ component of the stress tensor and $\zeta_{s}$ is the cooling rate. For a hydrodynamic description, $P_{x y, s}$ and $\zeta_{s}$ have to be expressed in terms of the hydrodynamic fields, $n_{s}, T_{s}$ and $\mathbf{u}_{s}$, and their gradients, i.e. the shear rate, $a[10,26]$. 
The steady USF state can be studied using kinetic theory. The definitions of the hydrodynamic fields in terms of the one-particle distribution function are those usually employed in kinetic theory

$$
\begin{aligned}
& n(\mathbf{r}, t) \equiv \int \mathrm{d} \mathbf{v} f_{1}(x, t), \\
& n(\mathbf{r}, t) \mathbf{u}(\mathbf{r}, t) \equiv \int \mathrm{d} \mathbf{v} \mathbf{v} f_{1}(x, t), \\
& \frac{d}{2} n(\mathbf{r}, t) T(\mathbf{r}, t) \equiv \int \mathrm{d} \mathbf{v} \frac{m}{2}[\mathbf{v}-\mathbf{u}(\mathbf{r}, t)]^{2} f_{1}(x, t) .
\end{aligned}
$$

The expressions for the pressure tensor and cooling rate are [10]

$$
P_{i j}(\mathbf{r}, t)=m \int \mathrm{d} \mathbf{v}\left[v_{i}-u_{i}(\mathbf{r}, t)\right]\left[v_{j}-u_{j}(\mathbf{r}, t)\right] f_{1}(x, t)
$$

and

$$
\zeta(\mathbf{r}, t)=\frac{\left(1-\alpha^{2}\right) \pi^{(d-1) / 2} m \sigma^{d-1}}{4 d \Gamma\left(\frac{d+3}{2}\right) n(\mathbf{r}, t) T(\mathbf{r}, t)} \int \mathrm{d} \mathbf{v}_{1} \int \mathrm{d} \mathbf{v}_{2} v_{12}^{3} f_{1}\left(\mathbf{r}, \mathbf{v}_{1}, t\right) f_{1}\left(\mathbf{r}, \mathbf{v}_{2}, t\right) .
$$

The Boltzmann equation admits a normal solution of the steady USF type, i.e. a solution in which all the space dependence arises from the hydrodynamic fields and all their gradients. As the only space-dependent field is linear, the distribution function can be written in a particularly simple form

$$
f_{\mathrm{USF}}(\mathbf{r}, \mathbf{v})=f_{s}\left[\mathbf{v}-\mathbf{u}_{s}(\mathbf{r}), n_{s}, T_{s}, a\right] .
$$

By substituting this expression into the Boltzmann equation, it follows that

$$
a V_{1 y} \frac{\partial}{\partial V_{1 x}} f_{s}\left(\mathbf{V}_{1}\right)+\int \mathrm{d} \mathbf{V}_{2} \bar{T}_{0}\left(\mathbf{V}_{1}, \mathbf{V}_{2}\right) f_{s}\left(\mathbf{V}_{1}\right) f_{s}\left(\mathbf{V}_{2}\right)=0
$$

where we have introduced the peculiar velocity $\mathbf{V} \equiv \mathbf{v}-\mathbf{u}_{s}(\mathbf{r})$, and we have omitted the explicit dependence on the hydrodynamic fields and the shear rate in the distribution function. Note that the form of the distribution given by equation (27) implies that the system is homogeneous in the Lagrangian frame of reference. Although the exact solution of equation (28) is not known, many approximate solutions are available [20-22, 24]. In this work we will consider the $\epsilon \equiv\left(1-\alpha^{2}\right)^{1 / 2}$ expansion of the Jenkins and Richman approximation up to order $\epsilon^{2}$. The specific form of the distribution will be given below.

It is convenient to perform the following change of variables

$$
\{\mathbf{r}, \mathbf{v}, t\} \longrightarrow\left\{\boldsymbol{\ell}(\mathbf{r}, t)=\mathbf{r}-a y t \hat{\mathbf{e}}_{x}, \mathbf{V}(\mathbf{r}, t)=\mathbf{v}-a y \hat{\mathbf{e}}_{x}, t\right\}
$$

The steady USF state is usually generated in particle simulations using Lees-Edwards boundary conditions [27] and, as stressed in [28], these become periodic boundary conditions in the new variables. As the Jacobian of the transformation is unity, the function

$$
f(\ell, \mathbf{V}, t)=f_{1}[\mathbf{r}(\ell, t), \mathbf{v}(\boldsymbol{\ell}, \mathbf{V}, t), t],
$$

is the actual distribution function in the new variables. Consider situations very close to the stationary USF state, in such a way that the deviations, $\delta f(\boldsymbol{\ell}, \mathbf{V}, t) \equiv f(\boldsymbol{\ell}, \mathbf{V}, t)-$ 
$f_{s}(\mathbf{V})$, are assumed to fulfill the condition $|\delta f(\boldsymbol{\ell}, \mathbf{V}, t)|<<f_{s}(\mathbf{V})$. To linear order, $\delta f$ satisfies

where

$$
\frac{\partial}{\partial t} \delta f\left(\boldsymbol{\ell}, \mathbf{V}_{1}, t\right)=H\left(\boldsymbol{\ell}, \mathbf{V}_{1}, t\right) \delta f\left(\boldsymbol{\ell}, \mathbf{V}_{1}, t\right)
$$

$$
H\left(\boldsymbol{\ell}, \mathbf{V}_{1}, t\right) \equiv L\left(\mathbf{V}_{1}\right)-\mathbf{V}_{1} \cdot \frac{\partial}{\partial \boldsymbol{\ell}}-a \ell_{y} \frac{\partial}{\partial \ell_{x}}+a t V_{1 y} \frac{\partial}{\partial \ell_{x}}
$$

is an inhomogeneous linear operator with

$L\left(\mathbf{V}_{1}\right) h\left(\mathbf{V}_{1}\right) \equiv \int \mathrm{d} \mathbf{V}_{2} \bar{T}_{0}\left(\mathbf{V}_{1}, \mathbf{V}_{2}\right)\left(1+P_{12}\right) f_{s}\left(\mathbf{V}_{1}\right) h\left(\mathbf{V}_{2}\right)+a V_{1 y} \frac{\partial}{\partial V_{1 x}} h\left(\mathbf{V}_{1}\right)$

Note that, in contrast with the free cooling case [11-13], the inhomogeneous term in equation (32) is time dependent.

Consider now the one-time and two-time correlation functions in the steady USF state, $g_{2 \mathrm{USF}}$ and $h_{1,1 \mathrm{USF}}$ respectively. In the new variables, the equations for

$$
G_{s}\left(\boldsymbol{\ell}_{1}, \mathbf{V}_{1}, \ell_{2}, \mathbf{V}_{2}\right) \equiv g_{2 \mathrm{USF}}\left(x_{1}, x_{2}\right)
$$

and

$$
h_{s}\left(\ell_{1}, \mathbf{V}_{1}, t ; \ell_{2}, \mathbf{V}_{2}, t^{\prime}\right) \equiv h_{1,1 \mathrm{USF}}\left(x_{1}, t ; x_{2}, t^{\prime}\right)
$$

are

$$
\left[H\left(\boldsymbol{\ell}_{1}, \mathbf{V}_{1}, t\right)+H\left(\boldsymbol{\ell}_{2}, \mathbf{V}_{2}, t\right)\right] G_{s}\left(\boldsymbol{\ell}_{1}, \mathbf{V}_{1}, \boldsymbol{\ell}_{2}, \mathbf{V}_{2}\right)=-\delta\left(\boldsymbol{\ell}_{12}\right) \bar{T}_{0}\left(\mathbf{V}_{1}, \mathbf{V}_{2}\right) f_{s}\left(\mathbf{V}_{1}\right) f_{s}\left(\mathbf{V}_{2}\right)
$$

and

$$
\frac{\partial}{\partial t} h_{s}\left(\boldsymbol{\ell}_{1}, \mathbf{V}_{1}, t ; \boldsymbol{\ell}_{2}, \mathbf{V}_{2}, t^{\prime}\right)=H\left(\boldsymbol{\ell}_{1}, \mathbf{V}_{1}, t\right) h_{s}\left(\boldsymbol{\ell}_{1}, \mathbf{V}_{1}, t ; \boldsymbol{\ell}_{2}, \mathbf{V}_{2}, t^{\prime}\right)
$$

respectively. This last equation has to be solved with the initial condition (see equation (19))

$$
h_{s}\left(\boldsymbol{\ell}_{1}, \mathbf{V}_{1}, t^{\prime} ; \boldsymbol{\ell}_{2}, \mathbf{V}_{2}, t^{\prime}\right)=f_{s}\left(\mathbf{V}_{1}\right) \delta\left(\boldsymbol{\ell}_{12}\right) \delta\left(\mathbf{V}_{12}\right)+G_{s}\left(\boldsymbol{\ell}_{1}, \mathbf{V}_{1}, \boldsymbol{\ell}_{2}, \mathbf{V}_{2}\right)
$$

Equations (36) and (37) describe one- and two-time two-particle correlations. Basically, they depend on the one-particle distribution function, which is assumed to be known, and on the linear operator defined by equation (33).

\subsection{Global correlations}

As the general problem is quite complex, we will focus on a simplified problem: the study of the correlations between global quantities. In order to deal with dimensionless distributions, we introduce the dimensionless velocity

$$
\mathbf{c}=\frac{\mathbf{V}}{v_{s}}, \quad v_{s}=\sqrt{\frac{2 T_{s}}{m}},
$$

through the thermal velocity in the stationary USF state, $v_{s}$, and the dimensionless time

$$
s=\frac{v_{s}}{\lambda} t, \quad \lambda=\left(n_{s} \sigma^{d-1}\right),
$$

with $\lambda$ proportional to the mean free path. In terms of these units, we define the dimensionless distributions. The scaled one-particle distribution function in the steady USF state is

$$
\chi(\mathbf{c}) \equiv \frac{v_{s}^{d}}{n_{s}} f_{s}(\mathbf{V}),
$$


the integrated deviation of the one-particle distribution function around the steady USF state is

$$
\delta \chi(\mathbf{c}, s) \equiv \frac{v_{s}^{d}}{n_{s}} \int \mathrm{d} \boldsymbol{\ell} \delta f(\boldsymbol{\ell}, \mathbf{V}, t),
$$

the dimensionless marginal one-time correlation function is

$$
\phi\left(\mathbf{c}_{1}, \mathbf{c}_{2}\right) \equiv \frac{v_{s}^{2 d}}{N} \int \mathrm{d} \boldsymbol{\ell}_{1} \int \mathrm{d} \boldsymbol{\ell}_{2} G_{s}\left(\boldsymbol{\ell}_{1}, \mathbf{V}_{1}, \boldsymbol{\ell}_{2}, \mathbf{V}_{2}\right)
$$

and the dimensionless marginal two-time correlation function is

$$
\psi\left(\mathbf{c}_{1}, \mathbf{c}_{2}, s-s^{\prime}\right) \equiv \frac{v_{s}^{2 d}}{N} \int \mathrm{d} \boldsymbol{\ell}_{1} \int \mathrm{d} \boldsymbol{\ell}_{2} h_{s}\left(\boldsymbol{\ell}_{1}, \mathbf{V}_{1}, t ; \boldsymbol{\ell}_{2}, \mathbf{V}_{2}, t^{\prime}\right) .
$$

For homogeneous states in the Lagrangian frame of reference, the evolution equation for $\delta \chi$, obtained by integrating equation (31), reads

$$
\frac{\partial}{\partial s} \delta \chi(\mathbf{c}, s)=\Lambda(\mathbf{c}) \delta \chi(\mathbf{c}, s)
$$

The operator $\Lambda$ will be called the linearized Boltzmann operator which is a dimensionless form of the linear operator defined in equation (33), i.e.

$$
\Lambda\left(\mathbf{c}_{1}\right) h\left(\mathbf{c}_{1}\right) \equiv \int \mathrm{d} \mathbf{c}_{2} \widetilde{T}_{0}\left(\mathbf{c}_{1}, \mathbf{c}_{2}\right)\left(1+P_{12}\right) \chi\left(\mathbf{c}_{1}\right) h\left(\mathbf{c}_{2}\right)+\tilde{a}_{s} c_{1 y} \frac{\partial}{\partial c_{1 x}} h\left(\mathbf{c}_{1}\right),
$$

where $\widetilde{T}_{0}$ is the dimensionless counterpart of $\bar{T}_{0}$

$$
\widetilde{T}_{0}\left(\mathbf{c}_{1}, \mathbf{c}_{2}\right) \equiv \int \mathrm{d} \hat{\boldsymbol{\sigma}} \Theta\left(\mathbf{c}_{12} \cdot \hat{\boldsymbol{\sigma}}\right)\left(\mathbf{c}_{12} \cdot \hat{\boldsymbol{\sigma}}\right)\left[\alpha^{-2} b_{\boldsymbol{\sigma}}^{-1}(1,2)-1\right]
$$

and

$$
\tilde{a}_{s}=\frac{\lambda a}{v_{s}}
$$

is the dimensionless shear rate.

The equation for the one-time correlation function is

$$
\left[\Lambda\left(\mathbf{c}_{1}\right)+\Lambda\left(\mathbf{c}_{2}\right)\right] \phi\left(\mathbf{c}_{1}, \mathbf{c}_{2}\right)=-\widetilde{T}_{0}\left(\mathbf{c}_{1}, \mathbf{c}_{2}\right) \chi\left(\mathbf{c}_{1}\right) \chi\left(\mathbf{c}_{2}\right),
$$

and the evolution equation for the two-time correlation functions is

$$
\frac{\partial}{\partial s} \psi\left(\mathbf{c}_{1}, \mathbf{c}_{2}, s\right)=\Lambda\left(\mathbf{c}_{1}\right) \psi\left(\mathbf{c}_{1}, \mathbf{c}_{2}, s\right),
$$

to be solved with the initial condition

$$
\psi\left(\mathbf{c}_{1}, \mathbf{c}_{2}, 0\right)=\chi\left(\mathbf{c}_{1}\right) \delta\left(\mathbf{c}_{12}\right)+\phi\left(\mathbf{c}_{1}, \mathbf{c}_{2}\right) .
$$

Note the strong analogy between equations (49) and (50), and the equivalents in other granular states (such as the homogeneous cooling state [9]), or other granular systems in which the particles are accelerated by a stochastic force [29]. The analogy is also evident with other dissipative systems [30], where the linearized Boltzmann collision operator, $\Lambda(\mathbf{c})$, always plays an essential role in the structure of the global correlations. 


\subsection{Correlations between global quantities}

Correlations between global quantities can be evaluated using the distributions we have introduced above. Consider quantities of the form

$$
\mathcal{A}(t)=\sum_{i=1}^{N} a\left[\mathbf{V}_{i}-\mathbf{u}_{s}\left(\mathbf{R}_{i}\right)\right]=\int \mathrm{d} \mathbf{r} \int \mathrm{d} \mathbf{v} a\left[\mathbf{v}-\mathbf{u}_{s}(\mathbf{r})\right] F_{1}(x, t),
$$

where $a$ is supposed to be a homogeneous function of degree $\beta$, i.e. $a(k \mathbf{c})=k^{\beta} a(\mathbf{c})$. The deviation around the mean in the steady USF state is

where

$$
\delta \mathcal{A}(t) \equiv \mathcal{A}(t)-\langle\mathcal{A}(t)\rangle=\int \mathrm{d} \mathbf{r} \int \mathrm{d} \mathbf{v} a(\mathbf{V}) \delta F(x, t),
$$

$$
\delta F(x, t) \equiv F_{1}(x, t)-f_{\mathrm{USF}}(x) .
$$

Correlations between the fluctuations of two different quantities, $\mathcal{A}_{1}$ and $\mathcal{A}_{2}$, of the form given in equation (52) can be expressed as

$\left\langle\delta \mathcal{A}_{1}(t) \delta \mathcal{A}_{2}\left(t^{\prime}\right)\right\rangle=\int \mathrm{d} \mathbf{r}_{1} \int \mathrm{d} \mathbf{v}_{1} \int \mathrm{d} \mathbf{r}_{2} \int \mathrm{d} \mathbf{v}_{2} a_{1}\left(\mathbf{V}_{1}\right) a_{2}\left(\mathbf{V}_{2}\right) h_{1,1 \mathrm{USF}}\left(x_{1}, t ; x_{2}, t^{\prime}\right)$.

In writing this expression, we have made use of the following:

$$
\left\langle\delta F\left(x_{1}, t\right) \delta F\left(x_{2}, t^{\prime}\right)\right\rangle=h_{1,1 \mathrm{USF}}\left(x_{1}, t ; x_{2}, t^{\prime}\right) .
$$

Expressing the integrand of (55) in terms of the dimensionless distribution defined in equation (44), yields

$$
\left\langle\delta \mathcal{A}_{1}(t) \delta \mathcal{A}_{2}\left(t^{\prime}\right)\right\rangle=N v_{s}^{\beta_{1}+\beta_{2}} \int \mathrm{d} \mathbf{c}_{1} \int \mathrm{d} \mathbf{c}_{2} a_{1}\left(\mathbf{c}_{1}\right) a_{2}\left(\mathbf{c}_{2}\right) \psi\left(\mathbf{c}_{1}, \mathbf{c}_{2}, s-s^{\prime}\right),
$$

where $\beta_{1}$ and $\beta_{2}$ are the degrees of homogeneity of $a_{1}$ and $a_{2}$, respectively.

The expression for the one-time correlations is obtained by making $s=s^{\prime}$ in equation (57). Taking into account equation (51), it follows that

$\left\langle\delta \mathcal{A}_{1}(t) \delta \mathcal{A}_{2}(t)\right\rangle=N v_{s}^{\beta_{1}+\beta_{2}}\left[\int \mathrm{d} \mathbf{c} a_{1}(\mathbf{c}) a_{2}(\mathbf{c}) \chi(\mathbf{c})+\int \mathrm{d} \mathbf{c}_{1} \int \mathrm{d} \mathbf{c}_{2} a_{1}\left(\mathbf{c}_{1}\right) a_{2}\left(\mathbf{c}_{2}\right) \phi\left(\mathbf{c}_{1}, \mathbf{c}_{2}\right)\right]$.

\section{Fluctuations of the total internal energy}

At a microscopic scale, the total internal energy is defined as

$$
\mathcal{E}(t)=\sum_{i=1}^{N} \frac{m}{2}\left[\mathbf{V}_{i}-\mathbf{u}_{s}\left(\mathbf{R}_{i}\right)\right]^{2}
$$

it is thus a quantity of the form introduced in the previous section. We identify, $a(\mathbf{V}) \equiv \frac{m}{2} V^{2}$, that is a homogeneous function of degree two. Using equation (58), we get

$$
\left\langle\delta \mathcal{E}^{2}(t)\right\rangle=\frac{m^{2}}{4} N v_{s}^{4}\left[\int \mathrm{d} \mathbf{c} c^{4} \chi(\mathbf{c})+\int \mathrm{d} \mathbf{c}_{1} \int \mathrm{d} \mathbf{c}_{2} c_{1}^{2} c_{2}^{2} \phi\left(\mathbf{c}_{1}, \mathbf{c}_{2}\right)\right] .
$$

Since the distribution $\chi$ is assumed to be known, we only have to evaluate the velocity moment of $\phi$ that appears in the right hand side of equation (60). To do this we follow a method based on the analysis of some spectral properties of the linearized Boltzmann collision operator, $\Lambda[9,29,30]$. 


\subsection{Spectral properties of $\Lambda$}

In order to identify some modes of the $\Lambda$ operator, it is necessary to introduce the timedependent USF state [31,32]. As for the stationary USF, the time-dependent state is characterized macroscopically by a constant density, $n_{H}$, and a time-independent flow velocity, $\mathbf{u}_{H}(\mathbf{r})=$ ay $\hat{\mathbf{e}}_{\mathbf{x}}$. The temperature, $T_{H}(t)$, remains homogeneous, but is timedependent. The subindex $H$ distinguishes it from the stationary state labelled as $s$. By dimensional analysis, if a normal distribution function for this state exists, it has the form

$$
f_{H}(\mathbf{V}, t)=\frac{n_{H}}{v_{H}(t)^{d}} \chi(\mathbf{c}, \tilde{a})
$$

where

$$
\mathbf{c}=\frac{\mathbf{V}}{v_{H}(t)}, \quad v_{H}(t)=\sqrt{\frac{2 T_{H}(t)}{m}}, \quad \tilde{a}=\frac{\lambda a}{v_{H}(t)} .
$$

We are using the same notation for the time-dependent scaled velocity $\mathbf{V} / v_{H}(t)$ and for $\mathbf{V} / v_{s}$ but this will not cause any difficulty. At long times, this distribution tends to the stationary one

$$
\chi(\mathbf{c}, \tilde{a}) \rightarrow \chi\left(\mathbf{c}, \tilde{a}_{s}\right) \equiv \chi(\mathbf{c}),
$$

and the quantities $v_{H}(t)$ and $\tilde{a}$ tend to their stationary values $v_{s}$ and $\tilde{a}_{s}$ respectively.

Consider the family of states given by equation (61) with the restriction of being close to the stationary USF state. These states are characterized by the two parameters

$$
\rho \equiv \frac{\delta n}{n_{s}}, \quad \theta \equiv \frac{\delta T}{T_{s}} .
$$

It is assumed that the deviations

$$
\delta n \equiv n_{H}-n_{s}, \quad \delta T \equiv T_{H}-T_{s},
$$

are small, i.e. $|\delta n| \ll n_{s}$ and $|\delta T| \ll T_{s}$. Global perturbations in the velocity field are not considered since we are always working in the frame of reference where total momentum is zero. We do not include states with different shear rates, $a$, because we want all the states to be generated by the same boundary conditions. Performing an analysis similar to that in reference [33], the following evolution equation for $\theta$

$$
\frac{\mathrm{d} \theta(s)}{\mathrm{d} s}=-\gamma[2 \rho+\theta(s)]
$$

is obtained in appendix A. As the total number of particles does not vary, $\rho$ is constant and we can identify the normal mode $[2 \rho+\theta(s)]$. The eigenvalue

$$
\gamma=\frac{\tilde{\zeta}\left(\tilde{a}_{s}\right)}{2}-\frac{\tilde{a}_{s}^{2}}{d} \frac{\mathrm{d} \tilde{P}_{x y}}{\mathrm{~d} \tilde{a}}\left(\tilde{a}_{s}\right)-\frac{\tilde{a}_{s}}{2} \frac{\mathrm{d} \tilde{\zeta}}{\mathrm{d} \tilde{a}}\left(\tilde{a}_{s}\right)
$$

is expressed in terms of the dimensionless pressure tensor

$$
\tilde{P}_{x y}(\tilde{a})=2 \int \mathrm{d} \mathbf{c} c_{x} c_{y} \chi(\mathbf{c}, \tilde{a})
$$

and the dimensionless cooling rate

$$
\tilde{\zeta}(\tilde{a})=\frac{\pi^{(d-1) / 2}\left(1-\alpha^{2}\right)}{2 \mathrm{~d} \Gamma\left(\frac{d+3}{2}\right)} \int \mathrm{d} \mathbf{c}_{1} \int \mathrm{d} \mathbf{c}_{2} c_{12}^{3} \chi\left(\mathbf{c}_{1}, \tilde{a}\right) \chi\left(\mathbf{c}_{2}, \tilde{a}\right),
$$

in the time-dependent USF state. Equation (67) is equivalent to that derived in [34] and to that of [33] for the case $\rho=0$. An explicit formula for $\gamma$ as a function of the inelasticity can be written using the expressions $\tilde{P}_{x y}$ and $\tilde{\zeta}\left(\tilde{a}_{s}\right)$ of the BGK model studied in [32], and neglecting the contribution proportional to $\mathrm{d} \tilde{\zeta} / \mathrm{d} \tilde{a}$. 


\subsection{An invalid approximation}

Let us rewrite equation (66) in a way that suggests the approximation to be analyzed in the following. Define the scalar product

$$
\langle h(\mathbf{c}) \mid g(\mathbf{c})\rangle \equiv \int \mathrm{d} \mathbf{c} h^{*}(\mathbf{c}) g(\mathbf{c})
$$

the star denoting the complex conjugate. The deviations $\rho$ and $\theta$ can be expressed in terms of $\delta \chi$ as

$$
\rho=\int \mathrm{d} \mathbf{c} \delta \chi(\mathbf{c}, s), \quad \theta(s)=\int \mathrm{d} \mathbf{c}\left(\frac{2}{d} c^{2}-1\right) \delta \chi(\mathbf{c}, s),
$$

and then

$$
2 \rho+\theta(s)=\int \mathrm{d} \mathbf{c}\left(\frac{2}{d} c^{2}+1\right) \delta \chi(\mathbf{c}, s) \equiv\left\langle\bar{\xi}_{2}(c) \mid \delta \chi(\mathbf{c}, s)\right\rangle,
$$

where we have introduced

$$
\bar{\xi}_{2}(c) \equiv \frac{2}{d} c^{2}+1
$$

By taking the scalar product with $\bar{\xi}_{2}$ in equation (45), one obtains

$$
\frac{\mathrm{d}}{\mathrm{d} s}\left\langle\bar{\xi}_{2}(c) \mid \delta \chi(\mathbf{c}, s)\right\rangle=\left\langle\bar{\xi}_{2}(c) \mid \Lambda(\mathbf{c}) \delta \chi(\mathbf{c}, s)\right\rangle .
$$

Comparing this equation with the evolution equation for $\theta$, equation (66), it is seen that, for $\delta \chi$ which belongs to the biparametric family of functions of time-dependent USF states that are closed to the stationary USF state, $\left\langle\bar{\xi}_{2}(c) \mid \Lambda(\mathbf{c}) \delta \chi(\mathbf{c}, s)\right\rangle=-\gamma\left\langle\bar{\xi}_{2}(\mathbf{c}) \mid \delta \chi(\mathbf{c}, s)\right\rangle$. It is consistent to consider the approximation

$$
\left\langle\bar{\xi}_{2}(c) \mid \Lambda(\mathbf{c}) g(\mathbf{c})\right\rangle \approx-\gamma\left\langle\bar{\xi}_{2}(\mathbf{c}) \mid g(\mathbf{c})\right\rangle,
$$

for any function, $g(\mathbf{c})$. This is, essentially, the approximation used to calculate the fluctuations of the total energy in $[9,29,30]$. Let us also mention that, in the free-cooling case, the equivalent of equation (75) is an exact property for Maxwell molecules [35].

However, it will be shown that equation (75), although consistent with linear hydrodynamics, is not consistent with the equation for $\phi$, equation (49). We initially see that some velocity moments of $\phi$ can be exactly related to velocity moments of the one-particle distribution, $\chi$. As the total number of particles, $N$, does not fluctuate, it is evident that

$$
\langle\delta N(t) \delta \mathcal{A}(t)\rangle=0
$$

for any fluctuating quantity, $\mathcal{A}$. If, in addition, $\mathcal{A}$ can be expressed as in equation (52), we have

$$
\langle\delta N \delta \mathcal{A}(t)\rangle=N v_{s}^{\beta}\left[\int \mathrm{d} \mathbf{c} a(\mathbf{c}) \chi(\mathbf{c})+\int \mathrm{d} \mathbf{c}_{1} \int \mathrm{d} \mathbf{c}_{2} a\left(\mathbf{c}_{1}\right) \phi\left(\mathbf{c}_{1}, \mathbf{c}_{2}\right)\right],
$$

and it can be concluded that

$$
\int \mathrm{d} \mathbf{c}_{1} \int \mathrm{d} \mathbf{c}_{2} a\left(\mathbf{c}_{1}\right) \phi\left(\mathbf{c}_{1}, \mathbf{c}_{2}\right)=-\int \mathrm{d} \mathbf{c} a(\mathbf{c}) \chi(\mathbf{c}),
$$

for any homogeneous function, $a(\mathbf{c})$, of degree $\beta$. With this result we can easily calculate the component

$$
\left\langle\bar{\xi}_{2}\left(c_{1}\right) \mid \phi\left(\mathbf{c}_{1}, \mathbf{c}_{2}\right)\right\rangle=-\int \mathrm{d} \mathbf{c}\left(\frac{2 c^{2}}{d}+1\right) \chi(\mathbf{c})=-2 .
$$


On the other hand, the integral can also be evaluated by taking the scalar product with $\bar{\xi}_{2}$ in the equation for $\phi$, equation (49), obtaining

$$
\left\langle\bar{\xi}_{2}\left(c_{1}\right) \mid \phi\left(\mathbf{c}_{1}, \mathbf{c}_{2}\right)\right\rangle=\frac{1}{\gamma} \int \mathrm{d} \mathbf{c}_{1} \int \mathrm{d} \mathbf{c}_{2}\left(\frac{2 c^{2}}{d}+1\right) \widetilde{T}_{0}\left(\mathbf{c}_{1}, \mathbf{c}_{2}\right) \chi\left(\mathbf{c}_{1}\right) \chi\left(\mathbf{c}_{2}\right)=-\frac{\tilde{\zeta}_{s}}{\gamma},
$$

where the expression of $\tilde{\zeta}$, equation (69), has been used, and we have introduced the notation $\tilde{\zeta}_{s} \equiv \tilde{\zeta}\left(\tilde{a}_{s}\right)$. It follows that the approximation $(75)$ is inconsistent with the equation for the correlation function, equation (49), because it predicts a result for $\left\langle\bar{\xi}_{2}\left(c_{1}\right) \mid \phi\left(\mathbf{c}_{1}, \mathbf{c}_{2}\right)\right\rangle$ which differs from the exact one given by equation (79). The approximation is not even valid in the elastic limit since $\gamma \sim \tilde{\zeta}$ in that limit. In fact, when $\left\langle\delta E^{2}\right\rangle$ is calculated using the approximate expression $\left\langle c_{1}^{2} c_{2}^{2} \mid \phi\left(\mathbf{c}_{1}, \mathbf{c}_{2}\right)\right\rangle$ (evaluated using equation (75)), the result disagrees with those of [18] even for $\alpha \rightarrow 1$. This is not surprising, since the approximation is not valid in the elastic limit either.

\subsection{A valid approximation}

As the previous result is clearly unsatisfactory, it would be desirable to find an approximation that is consistent with both linear hydrodynamics and the equation for the correlation function. Note also that the linearized Boltzmann operator, $\Lambda$, given by equation (46), contains a term of the form $c_{y} \partial / \partial c_{x}$ which mixes the subspace generated by $c^{2}$ with $c_{x} c_{y}$. It cannot therefore be expected that $\left\langle\bar{\xi}_{2}\right| \Lambda \approx-\gamma\left\langle\bar{\xi}_{2}\right|$ is a good approximation in general. In fact, the operator $c_{y} \partial / \partial c_{x}$ leaves invariant the 4-dimensional subspace generated by $\left\{1, c^{2}, c_{x} c_{y}, c_{y}^{2}\right\}$ and, for Maxwell molecules, the left eigenfunctions of $\Lambda$ are linear combinations of these 4 functions [36]. With this in mind, we will seek a generalization of approximation (75) taking as a possible candidate for $\bar{\xi}_{2}$ a function in the subspace generated by $\left\{1, c^{2}, c_{x} c_{y}, c_{y}^{2}\right\}$. To identify it, we consider the evolution equations for the homogeneous pressure tensor components of references $[22,24]$

$$
\begin{aligned}
& \frac{\partial T_{H}}{\partial t}+\zeta_{H} T_{H}+\frac{2 a}{d n_{H}} P_{x y, H}=0, \\
& \frac{\partial P_{x y, H}}{\partial t}+\left(\beta \nu_{H}+\zeta_{H}\right) P_{x y, H}+a P_{y y, H}=0, \\
& \frac{\partial P_{y y, H}}{\partial t}+\left(\beta \nu_{H}+\zeta_{H}\right) P_{y y, H}-\beta n_{H} \nu_{H} T_{H}=0,
\end{aligned}
$$

where we have introduced the subindex $H$ to indicate that we are considering only homogeneous situations. The cooling rate can be expressed as

$$
\zeta_{H}=\frac{v_{H}}{\lambda} \tilde{\zeta}_{s}, \quad \tilde{\zeta}_{s}=\frac{\sqrt{2} \pi^{(d-1) / 2}\left(1-\alpha^{2}\right)}{d \Gamma(d / 2)}
$$

where $\tilde{\zeta}_{s}$ coincides with $\tilde{\zeta}\left(\tilde{a}_{s}\right)$ calculated in the Jenkins and Richman approximation to $\epsilon^{2}$ order, $\nu_{H}$ is the collision frequency

$$
\nu_{H}=\frac{v_{H}}{\lambda} z, \quad z=\frac{8 \pi^{(d-1) / 2}}{\sqrt{2}(d+2) \Gamma(d / 2)},
$$

and $\beta$ is a parameter to be specified later on. 
Equations (81)-(83) admit a stationary solution. Defining the dimensionless components of the pressure tensor in the stationary state

$$
\tilde{P}_{i j, s} \equiv \frac{P_{i j, s}}{n_{s} T_{s}}
$$

one obtains $[22,24]$

$$
\tilde{P}_{x y, s}=-\frac{\mathrm{d} \tilde{\zeta}_{s}}{2 \tilde{a}_{s}}, \quad \tilde{P}_{y y, s}=\frac{\beta}{\beta+\frac{\tilde{\zeta}_{s}}{z}} .
$$

The dimensionless shear rate is

$$
\tilde{a}_{s}=z \sqrt{\frac{\mathrm{d} \tilde{\zeta}_{s}}{2 z \beta}}\left(\beta+\frac{\tilde{\zeta}_{s}}{z}\right),
$$

from which the stationary temperature can be evaluated through $v_{s}=\lambda a / \tilde{a}_{s}$. Note that all the expressions can be expressed in terms of $\tilde{\zeta}_{s}$ and $\beta$.

The set of equations (81)-(83), plus the trivial equation for the total density, can be linearized around the stationary state characterized by $n_{s}, T_{s}$ and $P_{i j, s}$. Defining the dimensionless deviations of the pressure tensor as

$$
\Pi_{i j}=\frac{P_{i j}-P_{i j, s}}{n_{s} T_{s}}
$$

we obtain the following set of linear equations

$$
\frac{d}{\partial s} \mathbf{y}(s)+M \mathbf{y}(s)=0
$$

for

$$
\mathbf{y}=\left[\begin{array}{c}
\rho(s) \\
\theta(s) \\
\Pi_{x y}(s) \\
\Pi_{y y}(s)
\end{array}\right]
$$

where we have introduced the matrix

$$
M=\left[\begin{array}{cccc}
0 & 0 & 0 & 0 \\
2 \tilde{\zeta}_{s} & \frac{3}{2} \tilde{\zeta}_{s} & \left(\beta z+\tilde{\zeta}_{s}\right) \sqrt{\frac{2 \tilde{\zeta}_{s}}{\mathrm{~d} \beta z}} & 0 \\
-\sqrt{\frac{\mathrm{d} \tilde{\zeta}_{s} \beta z}{2}} & -\frac{1}{2} \sqrt{\frac{\mathrm{d} \tilde{\zeta}_{s} \beta z}{2}} & \tilde{\zeta}_{s}+\beta z & \left(\beta z+\tilde{\zeta}_{s}\right) \sqrt{\frac{\mathrm{d} \tilde{\zeta}_{s}}{2 \beta z}} \\
-\beta z & -\beta z & 0 & \tilde{\zeta}_{s}+\beta z
\end{array}\right]
$$

that, again, is expressed in terms of $\tilde{\zeta}_{s}$ and $\beta$. Taking the explicit value of $\beta$ evaluated in Grad's approximation [24]

$$
\beta=\frac{1+\alpha}{2}\left[1-\frac{d-1}{2 d}(1-\alpha)\right],
$$

the matrix is expressed in terms of the inelasticity alone, $\alpha$. In this way, equation (89) becomes a set of linear differential equations for the deviations, $\mathbf{y}$, defined in equation (90), where all the coefficients of the matrix $M$ are known functions of the coefficient of normal 
restitution, $\alpha$. This is the generalization of equation (66) that we sought. The eigenvalues, $\left\{\lambda_{i}\right\}_{i=1}^{4}$, and their corresponding left eigenfunctions of $M,\left\{\mathbf{v}_{i}\right\}_{i=1}^{4}$, fulfill

$$
\mathbf{v}_{i} \cdot M=\lambda_{i} \mathbf{v}_{i},
$$

and can be calculated with Mathematica. As the expressions are very long, here we write only the expansion to $\epsilon^{4}$ order for $d=2$

$$
\begin{aligned}
& \lambda_{1}=0, \quad \lambda_{2} \approx \sqrt{\frac{\pi}{2}} \epsilon^{2}-\frac{3}{4} \sqrt{\frac{\pi}{2}} \epsilon^{4}, \\
& \lambda_{3} \approx\left(\sqrt{2 \pi}+\frac{1}{2} \sqrt{\frac{\pi}{2}} \epsilon^{2}+\frac{1}{4} \sqrt{\frac{\pi}{2}} \epsilon^{4}\right)-\imath\left(\sqrt{\pi} \epsilon+\frac{19}{64} \epsilon^{3}\right), \quad \lambda_{4}=\lambda_{3}^{*} .
\end{aligned}
$$

The corresponding left eigenfunctions to the same order are

$$
\begin{aligned}
& \mathbf{v}_{1}=(1,0,0,0), \\
& \mathbf{v}_{2} \approx\left(4-\frac{7 \epsilon^{2}}{2}+\frac{17 \epsilon^{4}}{4}, 2-\frac{11 \epsilon^{2}}{4}+\frac{23 \epsilon^{4}}{8},-\sqrt{2} \epsilon+\frac{11 \epsilon^{3}}{8 \sqrt{2}}, \epsilon^{2}\right), \\
& \mathbf{v}_{3} \approx\left(-1-\frac{\epsilon^{2}}{2}+\epsilon^{4},-1+\frac{\epsilon^{2}}{16}+\frac{67 \epsilon^{4}}{128}, \frac{\epsilon}{4 \sqrt{2}}+\frac{19 \epsilon^{3}}{64 \sqrt{2}}, 1\right) \\
&-\imath\left(\frac{3 \epsilon^{3}}{2 \sqrt{2}}, \frac{\epsilon}{2 \sqrt{2}}+\frac{75 \epsilon^{3}}{128 \sqrt{2}}, 1-\frac{\epsilon^{2}}{64}-\frac{1841 \epsilon^{4}}{8192}, 0\right), \\
& \mathbf{v}_{4}=\mathbf{v}_{3}^{*} .
\end{aligned}
$$

Note that, as equation (81) was the starting point for the derivation of equation (66), $\lambda_{2}$ can be expressed similarly to $\gamma$,

$$
\lambda_{2}=\frac{\tilde{\zeta}\left(\tilde{a}_{s}\right)}{2}-\frac{\tilde{a}_{s}^{2}}{d} \frac{\mathrm{d} \tilde{P}_{x y}}{\mathrm{~d} \tilde{a}}\left(\tilde{a}_{s}\right) .
$$

Here we do not have the $\frac{\mathrm{d} \tilde{\zeta}}{\mathrm{d} \tilde{a}}$ contribution since it was neglected from the outset.

With the aid of the left eigenfunctions, the normal modes of equation (89) can be easily written as

$$
\Xi_{j}=\mathbf{v}_{j} \cdot \mathbf{y}=v_{j 1} \rho+v_{j 2} \theta+v_{j 3} \Pi_{x y}+v_{j 4} \Pi_{y y},
$$

where $v_{j i}$ is the $i$-th component of $\mathbf{v}_{j}$. Now, we can identify the functions, $\left\{\bar{\xi}_{i}(\mathbf{c})\right\}_{i=1}^{4}$,

$$
\bar{\xi}_{i}(\mathbf{c})=\xi_{i 1}+\xi_{i 2} c^{2}+\xi_{i 3} c_{x} c_{y}+\xi_{i 4} c_{y}^{2},
$$

such that

$$
\left\langle\bar{\xi}_{j}(\mathbf{c}) \mid \delta \chi(\mathbf{c})\right\rangle=\Xi_{j} .
$$

Taking into account equation (71) and

$$
\Pi_{i j}(s)=2 \int \mathrm{d} \mathbf{c} c_{i} c_{j} \delta \chi(\mathbf{c}, s),
$$

we can identify

$$
\begin{aligned}
& \bar{\xi}_{1}(c)=1, \\
& \bar{\xi}_{2}(\mathbf{c})=\left(v_{21}-v_{22}\right)+\frac{2}{d} v_{22} c^{2}+2 v_{23} c_{x} c_{y}+2 v_{24} c_{y}^{2},
\end{aligned}
$$




$$
\begin{aligned}
& \bar{\xi}_{3}(\mathbf{c})=\left(v_{31}-v_{32}\right)+\frac{2}{d} v_{32} c^{2}+2 v_{33} c_{x} c_{y}+2 c_{y}^{2}, \\
& \bar{\xi}_{4}(\mathbf{c})=\bar{\xi}_{3}^{*}(\mathbf{c}) .
\end{aligned}
$$

Note that while the coefficients $\left\{v_{2 j}\right\}_{j=1}^{4}$ are real, $\left\{v_{3 j}\right\}_{j=1}^{3}$ have an imaginary part. Then the real and imaginary parts of $\lambda_{3}$ and $\bar{\xi}_{3}$ are introduced through

$$
\begin{aligned}
& \lambda_{3}=\lambda_{3}^{R}+\imath \lambda_{3}^{I}, \\
& \bar{\xi}_{3}(\mathbf{c})=\bar{\xi}_{3}^{R}(\mathbf{c})+\imath \bar{\xi}_{3}^{I}(\mathbf{c}) .
\end{aligned}
$$

In appendix B it is shown that the approximation

$$
\left\langle\bar{\xi}_{i}(\mathbf{c}) \mid \Lambda(\mathbf{c}) g(\mathbf{c})\right\rangle \approx-\lambda_{i}\left\langle\bar{\xi}_{i}(\mathbf{c}) \mid g(\mathbf{c})\right\rangle, \quad i=2,3,4 .
$$

is consistent with the equation for the correlation function, equation (49). Taking the scalar product with $\left\{\bar{\xi}_{i}\right\}_{i=1}^{3}$ in equation (49) an identity is obtained. Therefore, in contrast with approximation (75), the approximation given by equation (111) is fully valid, i.e. it is compatible with both linear hydrodynamics and the equation for the two-particle correlations.

To summarize, we have identified four modes. The first one (with the null eigenvalue) is trivial because it is that associated with the total number of particles. The second eigenvalue, $\lambda_{2}=\gamma$, vanishes in the elastic limit and is the one associated with the slowest excitations (at least in the elastic limit). For this reason, the second mode, $\Xi_{2}$, will be referred to in the following as the hydrodynamic mode. The last two modes (one is the complex conjugate of the other) decay faster and will be called kinetic modes. Note that, although we have extended the number of fields to describe the excitations of the system, the number of slow modes remains the same (i.e. we have not adopted a kind of extended hydrodynamics approach as could at first seem to be the case). Of course, these results are consistent with the ones of section 4.1. We obtain the same eigenvalue and, although the associated eigenfunctions are different, both modes are equivalent in the correct subspace. The differences in the modes are not important at the level of macroscopic hydrodynamics, but they are crucial at the level of two-particle correlations and, therefore, for identification of the correct fluctuating hydrodynamic equations [18].

Let us evaluate the fluctuations of the total energy using the approximation given by (111). This can be done by taking the scalar products with $\left\langle\bar{\xi}_{i}\left(\mathbf{c}_{1}\right) \bar{\xi}_{j}\left(\mathbf{c}_{2}\right)\right|$ in the equation (49) for $i, j=2,3,4$, but, in contrast with the previous cases, these fluctuations are coupled to those of the pressure tensor. We will nevertheless see that this coupling disappears in the elastic limit (restricting ourselves to $d=2$ ). In effect, multiplying equation (49) with $\left\langle\bar{\xi}_{2}\left(\mathbf{c}_{1}\right) \bar{\xi}_{2}\left(\mathbf{c}_{2}\right)\right|$ one obtains

$$
2 \gamma\left\langle\bar{\xi}_{2}\left(\mathbf{c}_{1}\right) \bar{\xi}_{2}\left(\mathbf{c}_{2}\right) \mid \phi\left(\mathbf{c}_{1}, \mathbf{c}_{2}\right)\right\rangle=-\left\langle\bar{\xi}_{2}\left(\mathbf{c}_{1}\right) \bar{\xi}_{2}\left(\mathbf{c}_{2}\right) \mid \widetilde{T}_{0}\left(\mathbf{c}_{1}, \mathbf{c}_{2}\right) \chi\left(\mathbf{c}_{1}\right) \chi\left(\mathbf{c}_{2}\right)\right\rangle,
$$

where approximation (111) has been used. For $d=2$ and for leading order terms only ( $\epsilon^{2}$ order in this case), we have

$$
\begin{gathered}
\gamma\left\langle\bar{\xi}_{2}\left(\mathbf{c}_{1}\right) \bar{\xi}_{2}\left(\mathbf{c}_{2}\right) \mid \phi\left(\mathbf{c}_{1}, \mathbf{c}_{2}\right)\right\rangle \approx \sqrt{\frac{\pi}{2}} \epsilon^{2}\left\langle\left(2+2 c_{1}^{2}\right)\left(2+2 c_{2}^{2}\right) \mid \phi\left(\mathbf{c}_{1}, \mathbf{c}_{2}\right)\right\rangle \\
=\sqrt{\frac{\pi}{2}} \epsilon^{2}\left[4\left\langle c_{1}^{2} c_{2}^{2} \mid \phi\left(\mathbf{c}_{1}, \mathbf{c}_{2}\right)\right\rangle-12\right] .
\end{gathered}
$$


The relation (78) has been used to evaluate

$$
\int \mathrm{d} \mathbf{c}_{1} \phi\left(\mathbf{c}_{1}, \mathbf{c}_{2}\right)=\int \mathrm{d} \mathbf{c}_{1} c_{i}^{2} \phi\left(\mathbf{c}_{1}, \mathbf{c}_{2}\right)=-1
$$

The right hand side of equation (112) is evaluated in appendix $\mathrm{C}$ using the $\epsilon$ expansion of the Jenkins and Richman approximation, obtaining

$$
\left\langle\bar{\xi}_{2}\left(\mathbf{c}_{1}\right) \bar{\xi}_{2}\left(\mathbf{c}_{2}\right) \mid \widetilde{T}_{0}\left(\mathbf{c}_{1}, \mathbf{c}_{2}\right) \chi\left(\mathbf{c}_{1}\right) \chi\left(\mathbf{c}_{2}\right)\right\rangle \approx-16 \sqrt{2 \pi} \epsilon^{2} .
$$

By introducing equations (113) and (115) into equation (112), we have

$$
\lim _{\alpha \rightarrow 1}\left\langle c_{1}^{2} c_{2}^{2} \mid \phi\left(\mathbf{c}_{1}, \mathbf{c}_{2}\right)\right\rangle=-1 .
$$

Finally, taking into account equation (60), we can calculate the elastic limit of

$$
N \frac{\left\langle\delta \mathcal{E}^{2}\right\rangle}{\langle\mathcal{E}\rangle^{2}}=\left[\int \mathrm{d} \mathbf{c} c^{4} \chi(\mathbf{c})+\int \mathrm{d} \mathbf{c}_{1} \int \mathrm{d} \mathbf{c}_{2} c_{1}^{2} c_{2}^{2} \phi\left(\mathbf{c}_{1}, \mathbf{c}_{2}\right)\right] \rightarrow 1,
$$

consistently with the results of [18].

\section{Fluctuations of the relevant global quantities}

The structure of the modes derived above implies a coupling between the fluctuations of the total energy and the fluctuations of the pressure tensor for finite $\epsilon$. In this section, all these cross correlations will be evaluated. The fluctuating total pressure tensor is defined as

$$
\mathcal{P}_{i j}(t) \equiv m \int \mathrm{d} \mathbf{r} \int \mathrm{d} \mathbf{v} V_{i} V_{j} F_{1}(x, t),
$$

and its deviation can be written in the form indicated in equation (53). The correlations between $\delta \mathcal{E}$ and $\delta \mathcal{P}_{i j}$ can be calculated with the aid of equation (58), obtaining

$$
\left\langle\delta \mathcal{E}(t) \delta \mathcal{P}_{i j}(t)\right\rangle=\frac{m^{2}}{2} N v_{s}^{4}\left[\int \mathrm{d} \mathbf{c} c^{2} c_{i} c_{j} \chi(\mathbf{c})+\int \mathrm{d} \mathbf{c}_{1} \int \mathrm{d} \mathbf{c}_{2} c_{1}^{2} c_{2 i} c_{2 j} \phi\left(\mathbf{c}_{1}, \mathbf{c}_{2}\right)\right]
$$

and analogously

$$
\left\langle\delta \mathcal{P}_{i j}(t) \delta \mathcal{P}_{n m}(t)\right\rangle=m^{2} N v_{s}^{4}\left[\int \mathrm{d} \mathbf{c} c_{i} c_{j} c_{n} c_{m} \chi(\mathbf{c})+\int \mathrm{d} \mathbf{c}_{1} \int \mathrm{d} \mathbf{c}_{2} c_{1 i} c_{1 j} c_{2 n} c_{2 m} \phi\left(\mathbf{c}_{1}, \mathbf{c}_{2}\right)\right] .
$$

This expression involves the first velocity moments of the correlation function, $\phi$. It is convenient to introduce the following notation

$$
\mathbf{b}(\mathbf{c})=\left[\begin{array}{c}
1 \\
c^{2} \\
c_{x} c_{y} \\
c_{y}^{2}
\end{array}\right],
$$

allowing the moments to be expressed in the following matrix form

$$
C_{i j}=\int \mathrm{d} \mathbf{c}_{1} \int \mathrm{d} \mathbf{c}_{2} b_{i}\left(\mathbf{c}_{1}\right) b_{j}\left(\mathbf{c}_{2}\right) \phi\left(\mathbf{c}_{1}, \mathbf{c}_{2}\right),
$$

that is trivially symmetric, i.e. $C_{i j}=C_{j i}$. 
The moments $\left\{C_{1 j}\right\}_{j=1}^{4}$ can easily be calculated because the total number of particles is conserved. Taking into account equation (78), we get

$$
C_{11}=-1, \quad C_{12}=-1, \quad C_{13}=-\frac{1}{2} \tilde{P}_{x y, s}, \quad C_{14}=-\frac{1}{2} \tilde{P}_{y y, s} .
$$

To calculate the other $C_{i j}$ the scalar product $\left\langle\bar{\xi}_{i}\left(\mathbf{c}_{1}\right) \bar{\xi}_{j}\left(\mathbf{c}_{2}\right)\right|$ is taken in equation (49) and the approximation (111) introduced, obtaining

$$
\left(\lambda_{i}+\lambda_{j}\right)\left\langle\bar{\xi}_{i}\left(\mathbf{c}_{1}\right) \bar{\xi}_{j}\left(\mathbf{c}_{2}\right) \mid \phi\left(\mathbf{c}_{1}, \mathbf{c}_{2}\right)\right\rangle=\left\langle\bar{\xi}_{i}\left(\mathbf{c}_{1}\right) \bar{\xi}_{j}\left(\mathbf{c}_{2}\right) \mid \widetilde{T}_{0}\left(\mathbf{c}_{1}, \mathbf{c}_{2}\right) \chi\left(\mathbf{c}_{1}\right) \chi\left(\mathbf{c}_{2}\right)\right\rangle, \quad i, j=2,3,4 .
$$

Actually, there are only 6 independent equations, because of the relation between the third and fourth modes. As the scalar products $\left\langle\bar{\xi}_{i}\left(\mathbf{c}_{1}\right) \bar{\xi}_{j}\left(\mathbf{c}_{2}\right) \mid \phi\left(\mathbf{c}_{1}, \mathbf{c}_{2}\right)\right\rangle$ (see equation (102) and (122)) can be written in terms of the $C_{i j}$ coefficients through

$$
\left\langle\bar{\xi}_{i}\left(\mathbf{c}_{1}\right) \bar{\xi}_{j}\left(\mathbf{c}_{2}\right) \mid \phi\left(\mathbf{c}_{1}, \mathbf{c}_{2}\right)\right\rangle=\sum_{l=1}^{4} \xi_{i l} \xi_{j l} C_{l l}+\sum_{k>l=1}^{4}\left(\xi_{i k} \xi_{j l}+\xi_{i l} \xi_{j k}\right) C_{k l},
$$

equation (124) defines a linear system of six equations for the six unknown coefficients $\left\{C_{22}, C_{23}, C_{24}, C_{33}, C_{34}, C_{44}\right\}$ (remember that $\left\{C_{1 j}\right\}_{j=1}^{4}$ are known).

The calculations leading to expressions for the coefficients $C_{i j}$ are detailed in appendix C. Since the $\left\langle\bar{\xi}_{i}\left(\mathbf{c}_{1}\right) \bar{\xi}_{j}\left(\mathbf{c}_{2}\right) \mid \widetilde{T}_{0}\left(\mathbf{c}_{1}, \mathbf{c}_{2}\right) \chi\left(\mathbf{c}_{1}\right) \chi\left(\mathbf{c}_{2}\right)\right\rangle$ are evaluated using the Jenkins and Richman distribution function for $d=2$, in the following all the results are restricted to this dimension. To order $\epsilon^{2}$ the expressions obtained are

$$
\begin{aligned}
C_{22}=-1+\frac{27}{32} \epsilon^{2}, \quad C_{23}=\frac{5}{8 \sqrt{2}} \epsilon, \quad C_{24}=-\frac{1}{2}+\frac{51}{64} \epsilon^{2}, \\
C_{33}=-\frac{23}{256} \epsilon^{2}, \quad C_{34}=\frac{5}{16 \sqrt{2}} \epsilon, \quad C_{44}=-\frac{1}{4}+\frac{145}{256} \epsilon^{2} .
\end{aligned}
$$

The one-particle averages that appear in the equations of the fluctuations, $\left\langle b_{i}(\mathbf{c}) b_{j}(\mathbf{c}) \mid \chi(\mathbf{c})\right\rangle$, can be calculated to the same approximation, obtaining

$$
\begin{aligned}
&\left\langle c^{4} \mid \chi(\mathbf{c})\right\rangle=2+ \frac{1}{2} \epsilon^{2}, \quad\left\langle c^{2} c_{x} c_{y} \mid \chi(\mathbf{c})\right\rangle=-\frac{3}{2 \sqrt{2}} \epsilon, \quad\left\langle c^{2} c_{y}^{2} \mid \chi(\mathbf{c})\right\rangle=1-\frac{1}{2} \epsilon^{2}, \\
&\left\langle c_{x}^{2} c_{y}^{2} \mid \chi(\mathbf{c})\right\rangle=\frac{1}{4}\left(1+\epsilon^{2}\right), \quad\left\langle c_{x} c_{y}^{3} \mid \chi(\mathbf{c})\right\rangle=-\frac{3}{4 \sqrt{2}} \epsilon, \quad\left\langle c_{y}^{4} \mid \chi(\mathbf{c})\right\rangle=\frac{3}{4}\left(1-\epsilon^{2}\right) .
\end{aligned}
$$

To express the final result in a compact notation it is useful to introduce the matrix elements

$$
B_{i j}(0) \equiv \int \mathrm{d} \mathbf{c}_{1} \int \mathrm{d} \mathbf{c}_{2} b_{i}\left(\mathbf{c}_{1}\right) b_{j}\left(\mathbf{c}_{2}\right) \psi\left(\mathbf{c}_{1}, \mathbf{c}_{2}, 0\right)=\left\langle b_{i}(\mathbf{c}) b_{j}(\mathbf{c}) \mid \chi(\mathbf{c})\right\rangle+C_{i j}
$$

By substituting equations (126) and (127) into the equation above, the expansion including terms up to $\epsilon^{2}$ of $B(0)$ is obtained,

$$
B(0)=\left[\begin{array}{cccc}
0 & 0 & 0 & 0 \\
0 & 1+\frac{199 \epsilon^{2}}{64} & -\frac{7 \epsilon}{8 \sqrt{2}} & \frac{1}{2}+\frac{19 \epsilon^{2}}{64} \\
0 & -\frac{7 \epsilon}{8 \sqrt{2}} & \frac{1}{4}+\frac{41 \epsilon^{2}}{256} & -\frac{7 \epsilon}{16 \sqrt{2}} \\
0 & \frac{1}{2}+\frac{19 \epsilon^{2}}{64} & -\frac{7 \epsilon}{16 \sqrt{2}} & \frac{1}{2}-\frac{47 \epsilon^{2}}{256}
\end{array}\right] .
$$


Finally, taking into account equations (128), (60), (119) and (120), we can express all the correlation functions in terms of $B_{i j}(0)$,

$$
\begin{aligned}
& \left\langle\delta \mathcal{E}^{2}(t)\right\rangle=\frac{m^{2}}{4} N v_{s}^{4} B_{22}(0), \quad\left\langle\delta \mathcal{E}(t) \delta \mathcal{P}_{x y}(t)\right\rangle=\frac{m^{2}}{2} N v_{s}^{4} B_{23}(0) \\
& \left\langle\delta \mathcal{E}(t) \delta \mathcal{P}_{y y}(t)\right\rangle=\frac{m^{2}}{2} N v_{s}^{4} B_{24}(0), \quad\left\langle\delta \mathcal{P}_{x y}^{2}(t)\right\rangle=m^{2} N v_{s}^{4} B_{33}(0) \\
& \left\langle\delta \mathcal{P}_{x y}(t) \delta \mathcal{P}_{y y}(t)\right\rangle=m^{2} N v_{s}^{4} B_{34}(0), \quad\left\langle\delta \mathcal{P}_{y y}^{2}(t)\right\rangle=m^{2} N v_{s}^{4} B_{44}(0)
\end{aligned}
$$

It is worth remarking that, although the system has been solved consistently to $\epsilon^{2}$ order, the expressions for the correlation functions are not the exact power expansion of the correlation functions. This is because the Jenkins and Richman approximation to $\epsilon^{2}$ order is not the exact expansion of the distribution [21].

Finally, let us calculate the two-time correlation functions between the global quantities already considered. Using equation (57) we arrive at the generalization of equations (60), (119) and (120) for two-times

$$
\begin{aligned}
& \left\langle\delta \mathcal{E}(t) \delta \mathcal{E}\left(t^{\prime}\right)\right\rangle=\frac{m^{2}}{4} N v_{s}^{4} \int \mathrm{d} \mathbf{c}_{1} \int \mathrm{d} \mathbf{c}_{2} c_{1}^{2} c_{2}^{2} \psi\left(\mathbf{c}_{1}, \mathbf{c}_{2} ; s-s^{\prime}\right) \\
& \left\langle\delta \mathcal{E}(t) \delta \mathcal{P}_{i j}\left(t^{\prime}\right)\right\rangle=\frac{m^{2}}{2} N v_{s}^{4} \int \mathrm{d} \mathbf{c}_{1} \int \mathrm{d} \mathbf{c}_{2} c_{1}^{2} c_{2 i} c_{2 j} \psi\left(\mathbf{c}_{1}, \mathbf{c}_{2}, s-s^{\prime}\right) \\
& \left\langle\delta \mathcal{P}_{i j}(t) \delta \mathcal{E}\left(t^{\prime}\right)\right\rangle=\frac{m^{2}}{2} N v_{s}^{4} \int \mathrm{d} \mathbf{c}_{1} \int \mathrm{d} \mathbf{c}_{2} c_{1 i} c_{1 j} c_{2}^{2} \psi\left(\mathbf{c}_{1}, \mathbf{c}_{2}, s-s^{\prime}\right) \\
& \left\langle\delta \mathcal{P}_{i j}(t) \delta \mathcal{P}_{n m}\left(t^{\prime}\right)\right\rangle=m^{2} N v_{s}^{4} \int \mathrm{d} \mathbf{c}_{1} \int \mathrm{d} \mathbf{c}_{2} c_{1 i} c_{1 j} c_{2 n} c_{2 m} \psi\left(\mathbf{c}_{1}, \mathbf{c}_{2}, s-s^{\prime}\right)
\end{aligned}
$$

Again, it is convenient to define the matrix elements

$$
B_{i j}(s) \equiv \int \mathrm{d} \mathbf{c}_{1} \int \mathrm{d} \mathbf{c}_{2} b_{i}\left(\mathbf{c}_{1}\right) b_{j}\left(\mathbf{c}_{2}\right) \psi\left(\mathbf{c}_{1}, \mathbf{c}_{2}, s\right)
$$

Inserting the formal expression of $\psi(s)$

$$
\psi\left(\mathbf{c}_{1}, \mathbf{c}_{2}, s\right)=\mathrm{e}^{s \Lambda\left(\mathbf{c}_{1}\right)}\left[\chi\left(\mathbf{c}_{1}\right) \delta\left(\mathbf{c}_{12}\right)+\phi\left(\mathbf{c}_{1}, \mathbf{c}_{2}\right)\right]
$$

into the above equations, and taking into account that the functions $\left\{b_{i}(\mathbf{c})\right\}_{i=1}^{4}$ can be written in terms of the functions $\left\{\vec{\xi}_{i}(\mathbf{c})\right\}_{i=1}^{4}$, the correlation functions can be evaluated explicitly by using the approximation (111), with the result

$$
B_{i j}(s)=\sum_{l=1}^{4} \sum_{l=1}^{4} Q_{i k}^{-1} Q_{k l} \mathrm{e}^{\lambda_{k} s} B_{l j}(0), \quad s>0 .
$$


Here we have introduced the matrix $Q$

$$
\left[\begin{array}{l}
\bar{\xi}_{1}(\mathbf{c}) \\
\bar{\xi}_{2}(\mathbf{c}) \\
\bar{\xi}_{3}(\mathbf{c}) \\
\bar{\xi}_{4}(\mathbf{c})
\end{array}\right]=Q \mathbf{b}(\mathbf{c})
$$

and its inverse, $Q^{-1}$, that can be identified through equation (102). Up to terms in $\epsilon^{2}$ we have

$$
Q=\left[\begin{array}{llll}
\xi_{11} & \xi_{12} & \xi_{13} & \xi_{14} \\
\xi_{21} & \xi_{22} & \xi_{23} & \xi_{24} \\
\xi_{31} & \xi_{32} & \xi_{33} & \xi_{34} \\
\xi_{41} & \xi_{42} & \xi_{43} & \xi_{44}
\end{array}\right] \approx\left[\begin{array}{cccc}
1 & 0 & 0 & 0 \\
2-\frac{3 \epsilon^{2}}{4} & 2-\frac{11 \epsilon^{2}}{4} & -2 \sqrt{2} \epsilon & 2 \epsilon^{2} \\
-\frac{9 \epsilon^{2}}{16} & -1+\frac{\epsilon^{2}}{16}-\imath \frac{\epsilon}{2 \sqrt{2}} & \frac{\epsilon}{2 \sqrt{2}}-2 \imath\left(1-\frac{\epsilon^{2}}{64}\right) & 2 \\
-\frac{9 \epsilon^{2}}{16} & -1+\frac{\epsilon^{2}}{16}+\imath \frac{\epsilon}{2 \sqrt{2}} & \frac{\epsilon}{2 \sqrt{2}}+2 \imath\left(1-\frac{\epsilon^{2}}{64}\right) & 2
\end{array}\right]
$$

Obviously, the correlation functions given by (137) fulfill the initial conditions. Moreover, they are a linear combination of the two modes $\lambda_{2}$ and $\lambda_{3}\left(\lambda_{4}=\lambda_{3}^{*}\right)$. The complete expressions for all the correlation functions are very lengthy and here we only write explicitly the expressions for the two-time autocorrelation function of the energy and pressure tensor,

$$
\langle\delta \mathcal{E}(t) \delta \mathcal{E}(0)\rangle=\frac{m^{2}}{4} N v_{s}^{4} B_{22}(s), \quad\left\langle\delta \mathcal{P}_{x y}(t) \delta \mathcal{P}_{x y}(0)\right\rangle=m^{2} N v_{s}^{4} B_{33}(s),
$$

with $B_{22}(s)$ and $B_{33}(s)$ given by equation (137), i.e.

$$
\begin{aligned}
& B_{22}(s)=\left(1+\frac{225 \epsilon^{2}}{128}\right) \mathrm{e}^{-\lambda_{2} s}-\frac{5 \epsilon^{2}}{8} \cos \left(\lambda_{3}^{I} s\right) \mathrm{e}^{-\lambda_{3}^{R} s} \\
& B_{33}(s)=\left[\left(\frac{1}{4}-\frac{3 \epsilon^{2}}{256}\right) \cos \left(\lambda_{3}^{I} s\right)+\frac{\sqrt{2} \epsilon}{32} \sin \left(\lambda_{3}^{I} s\right)\right] \mathrm{e}^{-\lambda_{3}^{R} s}+\frac{11 \epsilon^{2}}{64} \mathrm{e}^{-\lambda_{2} s} .
\end{aligned}
$$

We see that both functions have a hydrodynamic and a kinetic part. Nevertheless, the main contribution of $B_{22}(s)$ is the hydrodynamic one (the kinetic part is of order $\epsilon^{2}$ ), while the opposite occurs with $B_{33}(s)$.

\section{Simulation results}

We have performed Molecular Dynamics (MD) simulations of a two dimensional system of $N=2000$ inelastic hard disks of mass $m$ and diameter $\sigma$, in a square box of side $L$, corresponding to a number density $n_{s}=0.02 \sigma^{-2}$. To generate the stationary USF state, Lees-Edwards boundary conditions [27] in the $y$-direction and periodic boundary conditions in the $x$-direction have been used. Once the steady state was reached, we measured all the quantities studied in the previous section. The resulting values have been averaged over 300 trajectories, and in time over a period of about 150 collisions per particle. This has been done for various values of the inelasticity, $\alpha$. The shear rate was in all cases $a=6.32 \times 10^{-3}(T(0) / m)^{1 / 2} \sigma^{-1}$, where $T(0)$ is the initial temperature.

Figure 1 plots the quantity $B_{22}(0)$ as a function of the inelasticity. The symbols are the simulation results and the solid line the theoretical prediction given by equation (129). Also shown are the theoretical prediction of the fluctuating hydrodynamic approach (dashed line) and the improved one (dotted-line) taking into account rheological effects in 


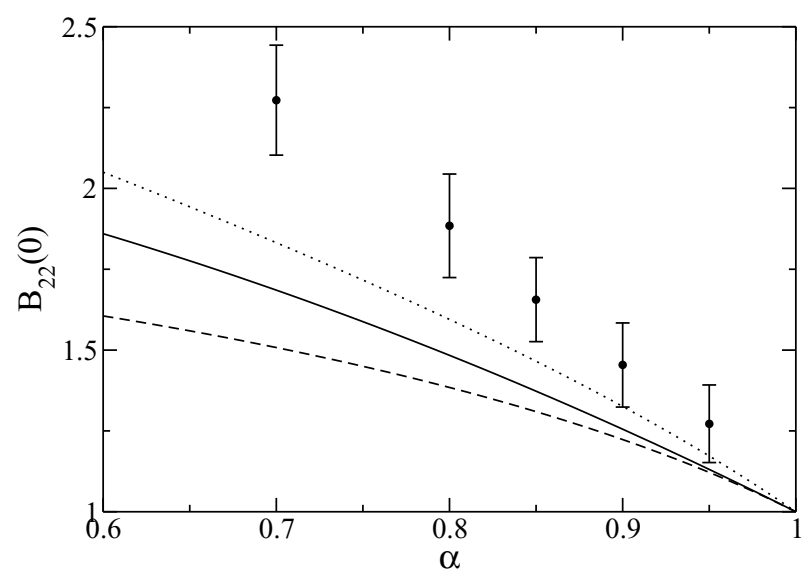

Figure 1. Dimensionless matrix element $B_{22}(0)$ as function of the restitution coefficient, $\alpha$, for a system of $N=2000$ hard disks. The symbols (dots) are the simulation results, the solid line is the theoretical prediction given by equation (129), the dashed line is the prediction using fluctuating hydrodynamics, and the dotted line the improved prediction given in [18].
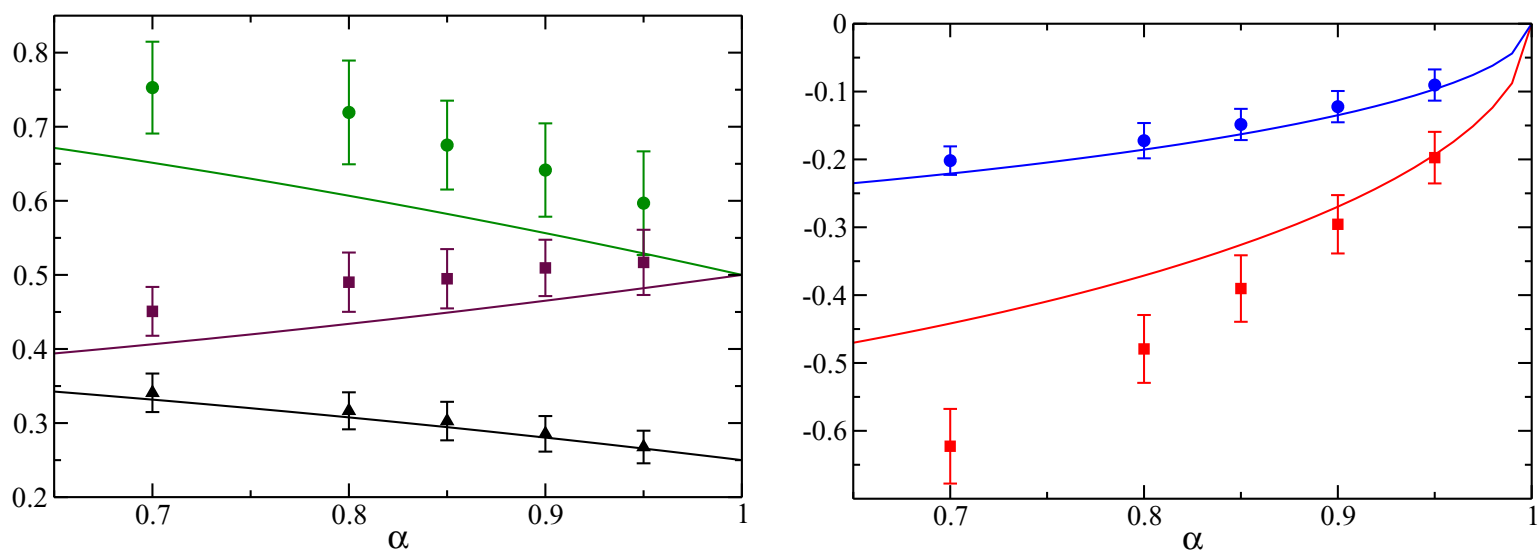

Figure 2. Dimensionless matrix elements of $B(0)$. The symbols are the simulation data and the solid lines correspond to the expansion to terms in $\epsilon^{2}$ given in equation (129). In the left figure, the circles, squares and triangles correspond to $B_{24}(0), B_{44}(0)$ and $B_{33}(0)$ respectively. In the right figure, the circles and squares correspond to $B_{34}(0)$ and $B_{23}(0)$ respectively.

the viscosity [18]. As the figure indicates, the last of these is very close to the prediction given by (129). Figure 2 plots the remaining matrix elements of $B$ as a function of $\alpha$. The solid lines are the theoretical predictions and the symbols are the simulation results. While the agreement is very good for $B_{23}(0), B_{33}(0)$ and $B_{34}(0)$, there are some discrepancies for $B_{22}(0), B_{24}(0)$ and $B_{44}(0)$ as the inelasticity increases.

As the $B_{i j}(0)$ coefficients have two components, the one-particle component and the correlation function component, we have measured the implied one-particle moments in order to establish the origin of the discrepancies. Figure 3 plots $\left\langle c^{4}\right\rangle$ and $\left\langle c^{2} c_{y}^{2}\right\rangle$. As the figure shows, there are important differences between the simulation results (points) and the Jenkins and Richman approximation (solid line). Also shown is the theoretical 
Fluctuations in the uniform shear flow state of a granular gas
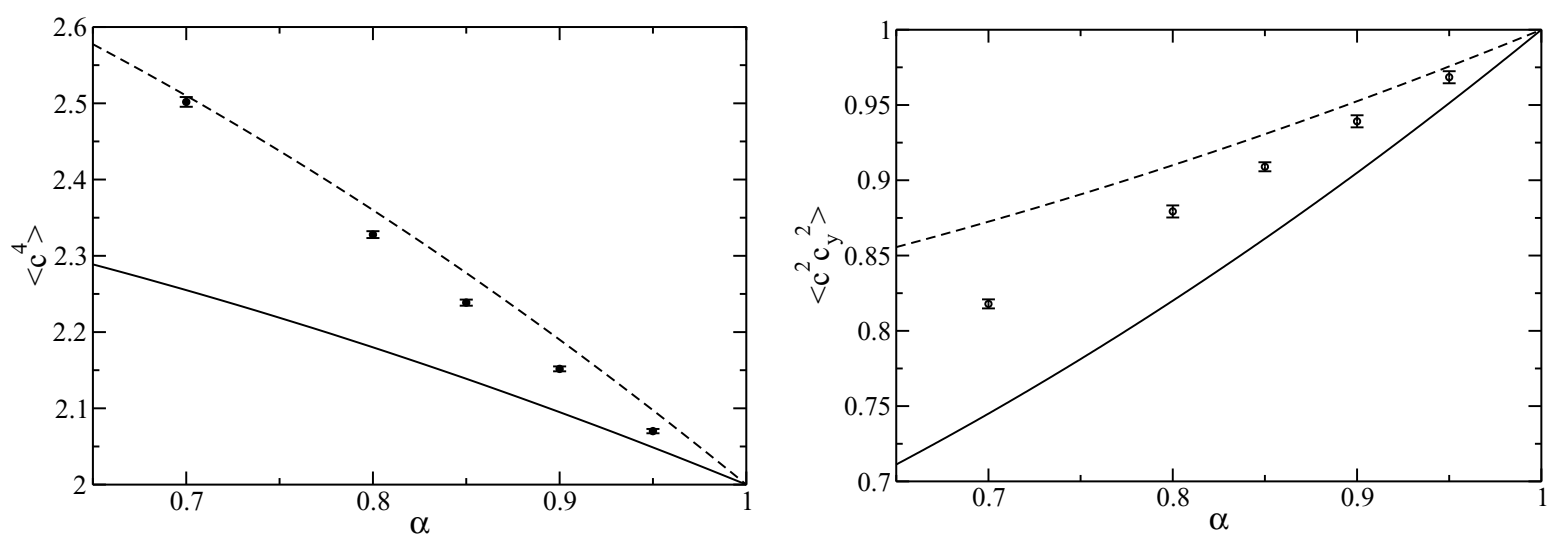

Figure 3. One-particle averages $\left\langle c^{4}\right\rangle$ (left) and $\left\langle c^{2} c_{y}^{2}\right\rangle$ (right). The simulation data (symbols) are compared with the predictions of Jenkins and Richman [20] (solid line) and with the BGK model [22] (dashed line).
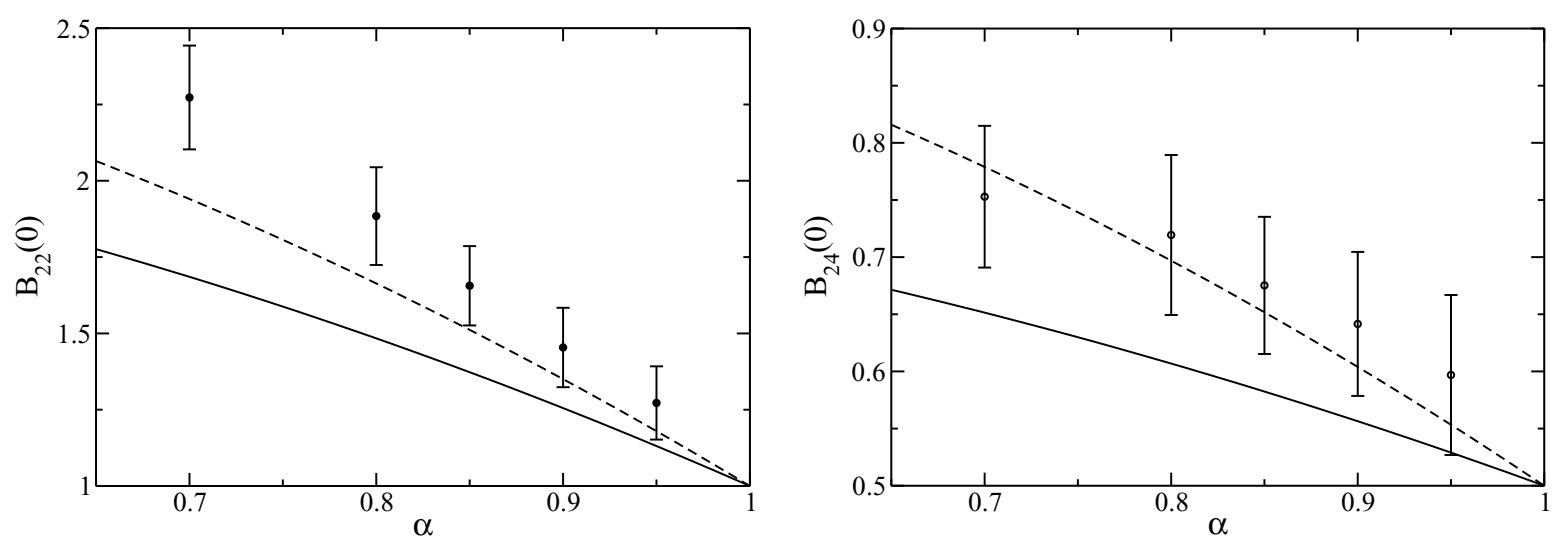

Figure 4. Comparison of the simulation results for $B_{22}(0)$ and $B_{24}(0)$ with the theoretical predictions using the Jenkins and Richman approximation [20] (solid lines) or the BGK model [22] (dashed line) for the one-particle moments.

prediction of the moments to terms in $\epsilon^{2}$ using the BGK model [22] which gives a much better agreement. Its explicit expressions are

$$
\left\langle c^{4}\right\rangle_{\mathrm{BGK}}=2+\epsilon^{2}, \quad\left\langle c^{2} c_{y}^{2}\right\rangle_{\mathrm{BGK}}=1-\frac{\epsilon^{2}}{4} .
$$

The remaining moments are accurately described by the Jenkins and Richman approximation. In fact, they coincide with the BGK ones apart from $\left\langle c_{x}^{2} c_{y}^{2}\right\rangle$, for which the Jenkins and Richman approximation does better than the BGK prediction.

Figure 4 plots $B_{22}(0)$ and $B_{24}(0)$ using the one-particle moments of the BGK model, showing that this considerably improves the agreement with the simulation results. Hence, we can conclude that agreement between the simulation results and the theoretical predictions (considering the most accurate expression for the one-particle moments) is excellent for all the coefficients, over the range of inelasticities considered. The only exception is $B_{44}$ for which the agreement is moderately good. We stress that, if the correlation function components are evaluated with the BGK distribution, the agreement is always worse than with the Jenkins and Richman one. 

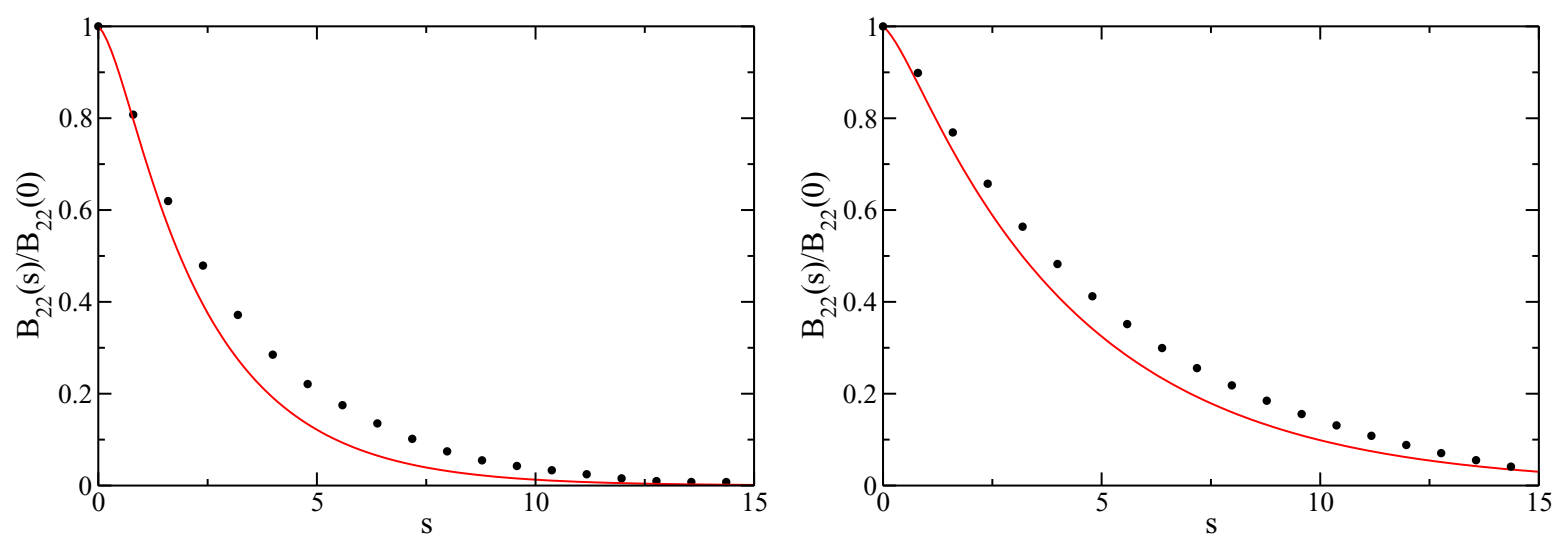

Figure 5. Decay of $B_{22}(s) / B_{22}(0)$ for a system with $\alpha=0.80$ (left) and $\alpha=0.90$ (right). The solid lines (red) are the predictions given by equation (141), and the symbols are the simulation results.
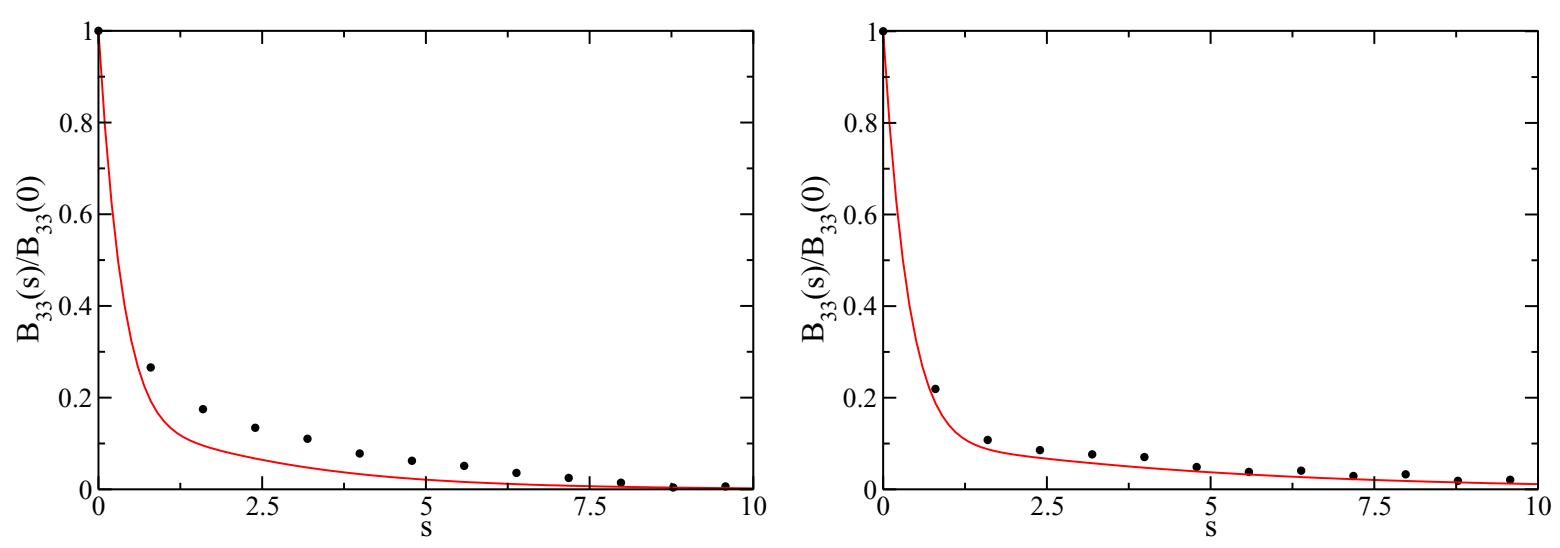

Figure 6. Decay of $B_{33}(s) / B_{33}(0)$ for a system with $\alpha=0.80$ (left) and $\alpha=0.90$ (right). The solid lines (red) are the predictions given by equation (142), and the symbols are the simulation results.

Finally, we have also measured the two-time correlation functions. Figure 5 shows the evolution of $B_{22}(s) / B_{22}(0)$, for systems with $\alpha=0.80$ (left) and $\alpha=0.90$ (right). In figure 6 the decay of $B_{33}(s) / B_{33}(0)$ has been plotted for the same values of the inelasticity. As the correlation functions are a combination of two exponentials, it is difficult to make a detailed comparison between the theoretical prediction and the simulation results. Nevertheless, we observe that $B_{33}(s)$ decays faster than $B_{22}(s)$, as predicted by equations (141) and (142). $B_{33}(s)$ has a hydrodynamic part that is of order $\epsilon^{2}$, while the kinetic part is of order unity (the contrary occurs for $B_{22}(s)$ ). We have also seen that, in the long time limit, both correlation functions decay in the same way (with the hydrodynamic mode). In order to see the behaviour of the functions at long times, they have been plotted on a logarithmic scale. This is done in figure 7 for a system with $\alpha=0.90$, where it is seen that, if the time $s$ is sufficiently large, the slopes become the same. 
Fluctuations in the uniform shear flow state of a granular gas

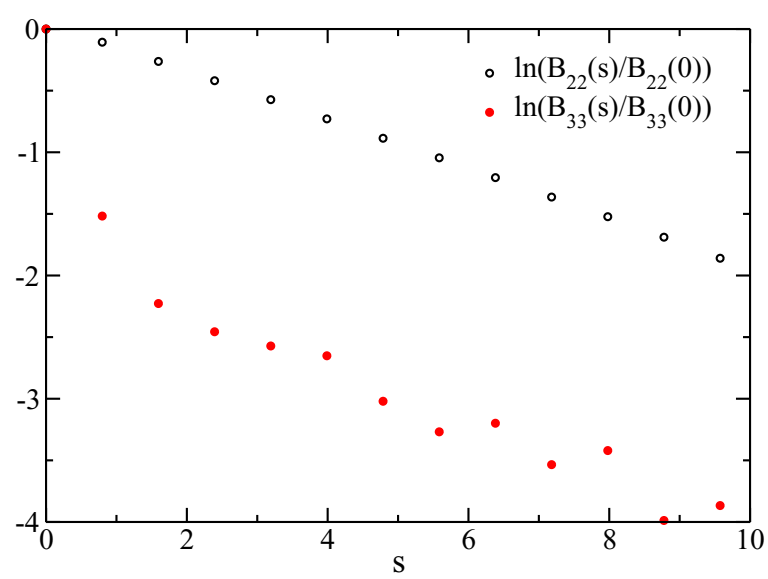

Figure 7. Comparison of the decay in the long time limit of the correlations $B_{22}(s) / B_{22}(0)$ and $B_{33}(s) / B_{33}(0)$ for a system with $\alpha=0.90$.

\section{Conclusion and discussion}

In this paper, we have studied the fluctuations of the total internal energy of a granular gas in the stationary USF state. Using the approximation given by equation (111), it has been shown that the fluctuations of the total internal energy are coupled to the fluctuations of the several components of the total pressure tensor. The approximation is fully consistent with the kinetic equation for the correlation function and is not, in principle, limited to small inelasticities. One of the main results of the paper is the closed system of equations given in (124), for the first six moments of the correlation function. With them, one can calculate all the possible one-time correlations of the total internal energy, and the different components of the total pressure tensor. The system depends on several complex moments of the one-particle distribution function in the stationary USF state. Since this distribution function is not known exactly, the Jenkins and Richman distribution has been used to $\epsilon^{2}$ order. For $d=2$, all the correlation functions have been evaluated as a function of the degree of inelasticity, $\epsilon$, giving a good agreement with Molecular Dynamics simulation results. The two-time correlations have also been evaluated.

At this point it is convenient to analyze the main analogies and differences between the HCS and the steady USF state. In both cases, there is no fluctuation-dissipation relation of the second kind, as the expression for the auto-correlation function of the total internal energy is not directly related to the coefficients of the macroscopic equation, which in this case is the cooling rate [18]. Moreover, in both the HCS state and the steady USF state, the two-time correlation function decays as a homogeneous macroscopic perturbation, see equation (50), so that there is a fluctuation-dissipation relation of the first kind. The main difference between the two cases lies in the nature of the approximation given in equation (111). While in the HCS case, the approximate eigenfunction can be identified by looking at the linearized homogeneous hydrodynamic equations, in the stationary USF case the equations for the pressure tensor components are needed. Although, in principle, this fact has no direct consequences at the level of macroscopic hydrodynamics, it is important at the level of the fluctuations. Actually, the correlation 
function, $\left\langle\delta \mathcal{P}_{x y}(t) \delta \mathcal{P}_{x y}(0)\right\rangle$, does not decay as a pure kinetic mode, as is the case in the HCS and as was assumed in [18]. The fluctuating quantity $\delta \mathcal{P}_{x y}(t)$ cannot thus be treated simply as a noise in a consistent way (one of the conditions for the results of [18] to hold was that the correlation function of the noise decays faster than that of the energy). We stress that, as the hydrodynamic part of the correlation function is of $\epsilon^{2}$ order, the coupling disappears in the elastic limit at which point we exactly recover the result of [18].

Finally, let us mention that many of the general properties shown in the paper can appear in any system beyond Navier-Stokes. Moreover, these results provide a starting point for the complete study of hydrodynamic fluctuating fields in the stationary USF state.

\section{Acknowledgments}

This research was supported by the Ministerio de Educación y Ciencia (Spain) through Grant No. FIS2011-24460 (partially financed by FEDER funds).

\section{Appendix A. Evolution equation for the temperature}

The objective of this appendix is to identify the mode that emerges after a homogeneous perturbation of the density and the temperature. Assuming that the hydrodynamic stage has been reached and the distribution function is that of the time-dependent USF state, we have

$$
\frac{\mathrm{d} T_{H}(t)}{\mathrm{d} t}=-\frac{2 a}{\mathrm{~d} n_{H}} P_{x y, H}(t)-\zeta_{H}(t) T_{H}(t)
$$

where the pressure tensor and cooling rate can be written as

$$
\begin{aligned}
& P_{x y, H}(t)=\frac{1}{2} n_{H} m v_{H}(t)^{2} \tilde{P}_{x y}(\tilde{a}), \\
& \zeta_{H}(t)=\frac{v_{H}(t)}{\lambda} \tilde{\zeta}(\tilde{a}),
\end{aligned}
$$

where $\tilde{P}_{x y}(\tilde{a})$ and $\tilde{\zeta}(\tilde{a})$ are defined in equations (68) and (69) respectively.

The deviation

$$
\delta\left[\frac{P_{x y, H}}{n_{H}}\right] \equiv \frac{P_{x y, H}}{n_{H}}-\frac{P_{x y, s}}{n_{s}},
$$

around the stationary value given by $n_{s}$ and $T_{s}$ is, to linear terms,

$$
\delta\left[\frac{P_{x y, H}}{n_{H}}\right]=\frac{1}{2} m v_{s}^{2} \tilde{P}_{x y, s} \theta-\frac{1}{2} m v_{s}^{2} \tilde{a}_{s} \frac{\mathrm{d} \tilde{P}_{x y}}{\mathrm{~d} \tilde{a}}\left(\tilde{a}_{s}\right)\left(\frac{1}{2} \theta+\rho\right),
$$

where we have used

$$
\left[\frac{\partial \tilde{a}}{\partial v_{H}}\right]_{v_{H}=v_{s}}=-\frac{\tilde{a}_{s}}{v_{s}}, \quad\left[\frac{\partial \tilde{a}}{\partial n_{H}}\right]_{v_{H}=v_{s}}=-\frac{\tilde{a}_{s}}{n_{s}} .
$$

By analogy, for the cooling rate term we have

$\delta\left[\zeta_{H}(t) T_{H}(t)\right]=T_{s} \frac{v_{s}}{\lambda}\left[\frac{3}{2} \tilde{\zeta}\left(\tilde{a}_{s}\right)-\frac{\tilde{a}_{s}}{2} \frac{\mathrm{d} \tilde{\zeta}}{\mathrm{d} \tilde{a}}\left(\tilde{a}_{s}\right)\right] \theta+T_{s} \frac{v_{s}}{\lambda}\left[\tilde{\zeta}\left(\tilde{a}_{s}\right)-\tilde{a}_{s} \frac{\mathrm{d} \tilde{\zeta}}{\mathrm{d} \tilde{a}}\left(\tilde{a}_{s}\right)\right] \rho$. 
Taking into account equations (A.5) and (A.7), we obtain

$$
\begin{aligned}
\frac{\mathrm{d} \theta}{\mathrm{d} s}=\left[2 \frac{\tilde{a}_{s}^{2}}{d} \frac{\partial \tilde{P}_{x y}}{\partial \tilde{a}}\left(\tilde{a}_{s}\right)-\tilde{\zeta}_{s}+\tilde{a}_{s} \frac{\mathrm{d} \tilde{\zeta}}{\mathrm{d} \tilde{a}}\left(\tilde{a}_{s}\right)\right] \rho & \\
+ & {\left[\frac{\tilde{a}_{s}^{2}}{d} \frac{\partial \tilde{P}_{x y}}{\partial \tilde{a}}\left(\tilde{a}_{s}\right)-\frac{2 \tilde{a}_{s}}{d} \tilde{P}_{x y, s}-\frac{3}{2} \tilde{\zeta}_{s}+\frac{1}{2} \tilde{a}_{s} \frac{\mathrm{d} \tilde{\zeta}}{\mathrm{d} \tilde{a}}\left(\tilde{a}_{s}\right)\right] \theta . }
\end{aligned}
$$

But, as in the stationary state we have

$$
\frac{2 \tilde{a}_{s}}{d} \tilde{P}_{x y, s}=-\tilde{\zeta}_{s}
$$

we obtain the result of the main text, equation (66).

\section{Appendix B. Validity of the approximation given by equation (111)}

In this Appendix we prove that the approximation given by equation (111) is consistent with the equation for the correlation function, equation (49). Taking the scalar product with $\bar{\xi}_{j}\left(\mathbf{c}_{1}\right)$ in equation (49) and performing the approximation (111), we obtain

$$
\lambda_{j}\left\langle\bar{\xi}_{j}\left(\mathbf{c}_{1}\right) \mid \phi\left(\mathbf{c}_{1}, \mathbf{c}_{2}\right)\right\rangle=\left\langle\bar{\xi}_{j}\left(\mathbf{c}_{1}\right) \mid \widetilde{T}_{0}\left(\mathbf{c}_{1}, \mathbf{c}_{2}\right) \chi\left(\mathbf{c}_{1}\right) \chi\left(\mathbf{c}_{2}\right)\right\rangle
$$

the validity of which can be proven. Here we will use a different approach. Equation (B.1) can be obtained by first integrating with respect to $\mathbf{c}_{2}$ in equation (49) and then taking the scalar product with $\bar{\xi}_{j}\left(\mathbf{c}_{1}\right)$ in the $\mathbf{c}_{1}$ space. The first step gives

$$
\Lambda(\mathbf{c}) \chi(\mathbf{c})=-\tilde{a}_{s} c_{y} \frac{\partial}{\partial c_{x}} \chi(\mathbf{c})
$$

where we have used

$$
\int \mathrm{d} \mathbf{c}_{2} \phi\left(\mathbf{c}_{1}, \mathbf{c}_{2}\right)=-\chi(\mathbf{c})
$$

Note that equation (B.2) is simply the non-linear Boltzmann equation for the stationary state, but expressed in terms of the linearized Boltzmann operator. Approximation (111) now has to be consistent when applied to equation (B.2), so that we have to prove that

$$
\lambda_{i}\left\langle\bar{\xi}_{i}(\mathbf{c}) \mid \chi(\mathbf{c})\right\rangle=\tilde{a}_{s}\left\langle\bar{\xi}_{i}(\mathbf{c}) \mid c_{y} \frac{\partial}{\partial c_{x}} \chi(\mathbf{c})\right\rangle, \quad i=2,3,4 .
$$

For $i=1$ the previous equation trivially holds.

Equations (B.4) can be expressed in a different basis of the subspace $\left\{\bar{\xi}_{i}\right\}_{i=1}^{4}$. The basis $\left\{h_{i}\right\}_{i=1}^{4}$ turns out to be useful, where

because we have

$$
\mathbf{h}(\mathbf{c})=\left[\begin{array}{c}
1 \\
\frac{2 c^{2}}{d}-1 \\
2 c_{x} c_{y} \\
2 c_{y}^{2}
\end{array}\right]
$$

$$
\int \mathrm{d} \mathbf{c h}(\mathbf{c}) \delta \chi(\mathbf{c}, s)=\left[\begin{array}{c}
\rho \\
\theta(s) \\
\Pi_{x y}(s) \\
\Pi_{y y}(s)
\end{array}\right]
$$


and, equation (B.4) can then be written as

$$
M\left[\begin{array}{c}
1 \\
0 \\
\tilde{P}_{x y, s} \\
\tilde{P}_{y y, s}
\end{array}\right]=-\tilde{a}_{s}\left[\begin{array}{c}
0 \\
\frac{2}{d} \tilde{P}_{x y, s} \\
\tilde{P}_{y y, s} \\
0
\end{array}\right]
$$

where we have used

$$
\int \mathrm{d} \mathbf{c h}(\mathbf{c}) \chi(\mathbf{c})=\left[\begin{array}{c}
1 \\
0 \\
\tilde{P}_{x y, s} \\
\tilde{P}_{y y, s}
\end{array}\right], \quad \int \mathrm{d} \mathbf{c h}(\mathbf{c}) \chi(\mathbf{c}) c_{y} \frac{\partial}{\partial c_{x}} \chi(\mathbf{c})=-\left[\begin{array}{c}
0 \\
\frac{2}{d} \tilde{P}_{x y, s} \\
\tilde{P}_{y y, s} \\
0
\end{array}\right] .
$$

Taking the explicit expressions of $M, \tilde{P}_{x y, s}$, and $\tilde{P}_{y y, s}$ as a function of $\tilde{\zeta}_{s}$ and $\beta$ of the main text, it is straightforward to prove the validity of equation (B.7).

\section{Appendix C. Evaluation of the $C_{i j}$ coefficients}

In this appendix we calculate the coefficients $C_{i j}$ defined in equation (122), starting from equation (124). As said, we only have 6 independent equations, because of the relation between the third and fourth mode. The corresponding equation for $i=j=2$ is

$$
2 \lambda_{2}\left\langle\bar{\xi}_{2}\left(\mathbf{c}_{1}\right) \bar{\xi}_{2}\left(\mathbf{c}_{2}\right) \mid \phi\left(\mathbf{c}_{1}, \mathbf{c}_{2}\right)\right\rangle=\left\langle\bar{\xi}_{2}\left(\mathbf{c}_{1}\right) \bar{\xi}_{2}\left(\mathbf{c}_{2}\right) \mid \widetilde{T}_{0}\left(\mathbf{c}_{1}, \mathbf{c}_{2}\right) \chi\left(\mathbf{c}_{1}\right) \chi\left(\mathbf{c}_{2}\right)\right\rangle .
$$

For $i=2$ and $j=3$ we have two equations, one associated with the real part

$$
\begin{gathered}
\left(\lambda_{2}+\lambda_{3}^{R}\right)\left\langle\bar{\xi}_{2}\left(\mathbf{c}_{1}\right) \bar{\xi}_{3}^{R}\left(\mathbf{c}_{2}\right) \mid \phi\left(\mathbf{c}_{1}, \mathbf{c}_{2}\right)\right\rangle-\lambda_{3}^{I}\left\langle\bar{\xi}_{2}\left(\mathbf{c}_{1}\right) \bar{\xi}_{3}^{I}\left(\mathbf{c}_{2}\right) \mid \phi\left(\mathbf{c}_{1}, \mathbf{c}_{2}\right)\right\rangle \\
=\left\langle\bar{\xi}_{2}\left(\mathbf{c}_{1}\right) \bar{\xi}_{3}^{R}\left(\mathbf{c}_{2}\right) \mid \widetilde{T}_{0}\left(\mathbf{c}_{1}, \mathbf{c}_{2}\right) \chi\left(\mathbf{c}_{1}\right) \chi\left(\mathbf{c}_{2}\right)\right\rangle,
\end{gathered}
$$

and other with the imaginary part

$$
\begin{gathered}
\left(\lambda_{2}+\lambda_{3}^{R}\right)\left\langle\bar{\xi}_{2}\left(\mathbf{c}_{1}\right) \bar{\xi}_{3}^{I}\left(\mathbf{c}_{2}\right) \mid \phi\left(\mathbf{c}_{1}, \mathbf{c}_{2}\right)\right\rangle-\lambda_{3}^{I}\left\langle\bar{\xi}_{2}\left(\mathbf{c}_{1}\right) \bar{\xi}_{3}^{R}\left(\mathbf{c}_{2}\right) \mid \phi\left(\mathbf{c}_{1}, \mathbf{c}_{2}\right)\right\rangle \\
=\left\langle\bar{\xi}_{2}\left(\mathbf{c}_{1}\right) \bar{\xi}_{3}^{I}\left(\mathbf{c}_{2}\right) \mid \widetilde{T}_{0}\left(\mathbf{c}_{1}, \mathbf{c}_{2}\right) \chi\left(\mathbf{c}_{1}\right) \chi\left(\mathbf{c}_{2}\right)\right\rangle,
\end{gathered}
$$

where we have used a decomposition into the real and imaginary parts of the third eigenvalue and eigenfunctions given by equations (109)-(110). For $i=j=3$ we also have two independent equations

$$
\begin{aligned}
2 \lambda_{3}^{R}\left[\left\langle\bar{\xi}_{3}^{R}\left(\mathbf{c}_{1}\right) \bar{\xi}_{3}^{R}\left(\mathbf{c}_{2}\right) \mid \phi\left(\mathbf{c}_{1}, \mathbf{c}_{2}\right)\right\rangle-\left\langle\bar{\xi}_{3}^{I}\left(\mathbf{c}_{1}\right) \bar{\xi}_{3}^{I}\left(\mathbf{c}_{2}\right) \mid \phi\left(\mathbf{c}_{1}, \mathbf{c}_{2}\right)\right\rangle\right]-4 \lambda_{3}^{I}\left\langle\bar{\xi}_{3}^{R}\left(\mathbf{c}_{1}\right) \bar{\xi}_{3}^{I}\left(\mathbf{c}_{2}\right) \mid \phi\left(\mathbf{c}_{1}, \mathbf{c}_{2}\right)\right\rangle \\
\quad=\left\langle\bar{\xi}_{3}^{R}\left(\mathbf{c}_{1}\right) \bar{\xi}_{3}^{R}\left(\mathbf{c}_{2}\right) \mid \widetilde{T}_{0}\left(\mathbf{c}_{1}, \mathbf{c}_{2}\right) \chi\left(\mathbf{c}_{1}\right) \chi\left(\mathbf{c}_{2}\right)\right\rangle \\
-\left\langle\bar{\xi}_{3}^{I}\left(\mathbf{c}_{1}\right) \bar{\xi}_{3}^{I}\left(\mathbf{c}_{2}\right) \mid \widetilde{T}_{0}\left(\mathbf{c}_{1}, \mathbf{c}_{2}\right) \chi\left(\mathbf{c}_{1}\right) \chi\left(\mathbf{c}_{2}\right)\right\rangle,
\end{aligned}
$$

and

$$
\begin{gathered}
\lambda_{3}^{I}\left[\left\langle\bar{\xi}_{3}^{R}\left(\mathbf{c}_{1}\right) \bar{\xi}_{3}^{R}\left(\mathbf{c}_{2}\right) \mid \phi\left(\mathbf{c}_{1}, \mathbf{c}_{2}\right)\right\rangle-\left\langle\bar{\xi}_{3}^{I}\left(\mathbf{c}_{1}\right) \bar{\xi}_{3}^{I}\left(\mathbf{c}_{2}\right) \mid \phi\left(\mathbf{c}_{1}, \mathbf{c}_{2}\right)\right\rangle\right]+2 \lambda_{3}^{R}\left\langle\bar{\xi}_{3}^{R}\left(\mathbf{c}_{1}\right) \bar{\xi}_{3}^{I}\left(\mathbf{c}_{2}\right) \mid \phi\left(\mathbf{c}_{1}, \mathbf{c}_{2}\right)\right\rangle \\
=\left\langle\bar{\xi}_{3}^{R}\left(\mathbf{c}_{1}\right) \bar{\xi}_{3}^{I}\left(\mathbf{c}_{2}\right) \mid \widetilde{T}_{0}\left(\mathbf{c}_{1}, \mathbf{c}_{2}\right) \chi\left(\mathbf{c}_{1}\right) \chi\left(\mathbf{c}_{2}\right)\right\rangle .
\end{gathered}
$$

Finally, there is an additional equation corresponding to $i=3, j=4$

$$
\begin{aligned}
\left.2 \lambda_{3}^{R}\left\langle\bar{\xi}_{3}^{R}\left(\mathbf{c}_{1}\right) \bar{\xi}_{3}^{R}\left(\mathbf{c}_{2}\right) \mid \phi\left(\mathbf{c}_{1}, \mathbf{c}_{2}\right)\right\rangle+\left\langle\bar{\xi}_{3}^{I}\left(\mathbf{c}_{1}\right) \bar{\xi}_{3}^{I}\left(\mathbf{c}_{2}\right) \mid \phi\left(\mathbf{c}_{1}, \mathbf{c}_{2}\right)\right\rangle\right] \\
=\left\langle\bar{\xi}_{3}^{R}\left(\mathbf{c}_{1}\right) \bar{\xi}_{3}^{R}\left(\mathbf{c}_{2}\right) \mid \widetilde{T}_{0}\left(\mathbf{c}_{1}, \mathbf{c}_{2}\right) \chi\left(\mathbf{c}_{1}\right) \chi\left(\mathbf{c}_{2}\right)\right\rangle \\
+\left\langle\bar{\xi}_{3}^{I}\left(\mathbf{c}_{1}\right) \bar{\xi}_{3}^{I}\left(\mathbf{c}_{2}\right) \mid \widetilde{T}_{0}\left(\mathbf{c}_{1}, \mathbf{c}_{2}\right) \chi\left(\mathbf{c}_{1}\right) \chi\left(\mathbf{c}_{2}\right)\right\rangle,
\end{aligned}
$$

that can be written in terms of the third mode because $\lambda_{4}=\lambda_{3}^{*}$ and $\bar{\xi}_{4}=\bar{\xi}_{3}^{*}$. 
The scalar products $\left\langle\bar{\xi}_{i}\left(\mathbf{c}_{1}\right) \bar{\xi}_{j}\left(\mathbf{c}_{2}\right) \mid \phi\left(\mathbf{c}_{1}, \mathbf{c}_{2}\right)\right\rangle$ can be written in terms of the $C_{i j}$ coefficients through equation (125), so that the system of equations (C.1)-(C.6) is a linear system of six equations for the six unknown coefficients $\left\{C_{22}, C_{23}, C_{24}, C_{33}, C_{34}, C_{44}\right\}$. Note that the results so far are valid for any dimension, $d$, and the only approximation made was that given by equation (111). Of course, it still remains to evaluate the coefficients

$$
\left\langle\bar{\xi}_{i}\left(\mathbf{c}_{1}\right) \bar{\xi}_{j}\left(\mathbf{c}_{2}\right) \mid \widetilde{T}_{0}\left(\mathbf{c}_{1}, \mathbf{c}_{2}\right) \chi\left(\mathbf{c}_{1}\right) \chi\left(\mathbf{c}_{2}\right)\right\rangle=\sum_{l=1}^{4} \xi_{i l} \xi_{j l} T_{l l}+\sum_{k>l=1}^{4}\left(\xi_{i k} \xi_{j l}+\xi_{i l} \xi_{j k}\right) T_{k l},
$$

where we have introduced the matrix elements

$$
\begin{aligned}
& T_{i j}=\int \mathrm{d} \mathbf{c}_{1} \int \mathrm{d} \mathbf{c}_{2} b_{i}\left(\mathbf{c}_{1}\right) b_{j}\left(\mathbf{c}_{2}\right) \widetilde{T}_{0}\left(\mathbf{c}_{1}, \mathbf{c}_{2}\right) \chi\left(\mathbf{c}_{1}\right) \chi\left(\mathbf{c}_{2}\right) \\
&=\int \mathrm{d} \mathbf{c}_{1} \int \mathrm{d} \mathbf{c}_{2} \chi\left(\mathbf{c}_{1}\right) \chi\left(\mathbf{c}_{2}\right) T_{0}\left(\mathbf{c}_{1}, \mathbf{c}_{2}\right) b_{i}\left(\mathbf{c}_{1}\right) b_{j}\left(\mathbf{c}_{2}\right),
\end{aligned}
$$

with

$$
T_{0}\left(\mathbf{c}_{1}, \mathbf{c}_{2}\right)=\int \mathrm{d} \hat{\boldsymbol{\sigma}} \Theta\left(\mathbf{c}_{12} \cdot \hat{\boldsymbol{\sigma}}\right)\left(\mathbf{c}_{12} \cdot \hat{\boldsymbol{\sigma}}\right)\left[b_{\boldsymbol{\sigma}}(1,2)-1\right]
$$

The first coefficients, $\left\{T_{1 j}\right\}_{j=1}^{4}$, can easily be calculated. In effect,

$$
T_{11}=0,
$$

due to the conservation of the total number of particles, and the second is related to the cooling rate

$$
T_{12}=-\frac{d}{2} \tilde{\zeta}_{s}
$$

by equation (69). On the other hand, taking into account the equation for $\chi$, equation (B.2), we have

$$
T_{13}=-\tilde{a}_{s} \int \mathrm{d} \mathbf{c} c_{x} c_{y}^{2} \frac{\partial}{\partial c_{x}} \chi(\mathbf{c})=\frac{1}{2} \tilde{a}_{s} \tilde{P}_{y y, s},
$$

and

$$
T_{14}=-\tilde{a}_{s} \int \mathrm{d} \mathbf{c} c_{y}^{2} \frac{\partial}{\partial c_{x}} \chi(\mathbf{c})=0 .
$$

To evaluate the other coefficients we have to calculate explicitly $T_{0}\left(\mathbf{c}_{1}, \mathbf{c}_{2}\right) b_{i}\left(\mathbf{c}_{1}\right) b_{j}\left(\mathbf{c}_{2}\right)$. In reference [9] the term $T_{0}\left(\mathbf{c}_{1}, \mathbf{c}_{2}\right) c_{1}^{2} c_{2}^{2}$ has already been calculated yielding

$$
\begin{aligned}
T_{0}\left(\mathbf{c}_{1}, \mathbf{c}_{2}\right) c_{1}^{2} c_{2}^{2}= & -\frac{\pi^{(d-1) / 2}}{\Gamma\left(\frac{d+5}{2}\right)}\left[\frac{\left(1-\alpha^{2}\right)\left(d+1+2 \alpha^{2}\right)}{16} g^{5}\right. \\
& \left.+\frac{d+5-\alpha^{2}(d+1)+4 \alpha}{4} g^{3} G^{2}-\frac{1+\alpha}{2}(2 d+3-3 \alpha) g(\mathbf{g} \cdot \mathbf{G})^{2}\right],
\end{aligned}
$$

where we have introduced the new variables

$$
\begin{aligned}
& \mathbf{g}=\mathbf{c}_{1}-\mathbf{c}_{2}, \\
& \mathbf{G}=\frac{1}{2}\left(\mathbf{c}_{1}+\mathbf{c}_{2}\right) .
\end{aligned}
$$


For the rest of coefficients, we first evaluate $\left[b_{\boldsymbol{\sigma}}(1,2)-1\right] b_{i}\left(\mathbf{c}_{1}\right) b_{j}\left(\mathbf{c}_{2}\right)$. Using the collision rule, equation (1), we obtain

$$
\begin{aligned}
{\left[b_{\boldsymbol{\sigma}}(1,2)-1\right] c_{1}^{2} c_{2 x} c_{2 y}=\frac{1+\alpha}{2}(\hat{\boldsymbol{\sigma}} \cdot \mathbf{g})\left[c_{1}^{2}\left(c_{2 x} \hat{\sigma}_{y}+c_{2 y} \hat{\sigma}_{x}\right)-2\left(\hat{\boldsymbol{\sigma}} \cdot \mathbf{c}_{1}\right) c_{2 x} c_{2 y}\right] } \\
+\frac{(1+\alpha)^{2}}{4}(\hat{\boldsymbol{\sigma}} \cdot \mathbf{g})^{2}\left[c_{1}^{2} \hat{\sigma}_{x} \hat{\sigma}_{y}+c_{2 x} c_{2 y}-2\left(\hat{\boldsymbol{\sigma}} \cdot \mathbf{c}_{1}\right)\left(c_{2 x} \hat{\sigma}_{y}+c_{2 y} \hat{\sigma}_{x}\right)\right] \\
+\frac{(1+\alpha)^{3}}{8}(\hat{\boldsymbol{\sigma}} \cdot \mathbf{g})^{3}\left[c_{2 x} \hat{\sigma}_{y}+c_{2 y} \hat{\sigma}_{x}-2\left(\hat{\boldsymbol{\sigma}} \cdot \mathbf{c}_{1}\right) \hat{\sigma}_{x} \hat{\sigma}_{y}\right]+\frac{(1+\alpha)^{4}}{16}(\hat{\boldsymbol{\sigma}} \cdot \mathbf{g})^{4} \hat{\sigma}_{x} \hat{\sigma}_{y},
\end{aligned}
$$

$$
\begin{aligned}
{\left[b_{\boldsymbol{\sigma}}(1,2)-1\right] } & c_{1}^{2} c_{2 y}^{2}=(1+\alpha)(\hat{\boldsymbol{\sigma}} \cdot \mathbf{g})\left[c_{1}^{2} c_{2 y} \hat{\sigma}_{y}-c_{2 y}^{2}\left(\hat{\boldsymbol{\sigma}} \cdot \mathbf{c}_{1}\right)\right] \\
& +\frac{(1+\alpha)^{2}}{4}(\hat{\boldsymbol{\sigma}} \cdot \mathbf{g})^{2}\left[c_{1}^{2} \hat{\sigma}_{y}^{2}+c_{2 y}^{2}-4\left(\hat{\boldsymbol{\sigma}} \cdot \mathbf{c}_{1}\right) c_{2 y} \hat{\sigma}_{y}\right] \\
& +\frac{(1+\alpha)^{3}}{4}(\hat{\boldsymbol{\sigma}} \cdot \mathbf{g})^{3}\left[c_{2 y} \hat{\sigma}_{y}-\left(\hat{\boldsymbol{\sigma}} \cdot \mathbf{c}_{1}\right) \hat{\sigma}_{y}^{2}\right]+\frac{(1+\alpha)^{4}}{16}(\hat{\boldsymbol{\sigma}} \cdot \mathbf{g})^{4} \hat{\sigma}_{y}^{2},
\end{aligned}
$$

$$
\left[b_{\boldsymbol{\sigma}}(1,2)-1\right] c_{1 x} c_{1 y} c_{2 x} c_{2 y}=\frac{1+\alpha}{2}(\hat{\boldsymbol{\sigma}} \cdot \mathbf{g})\left(\hat{\sigma}_{y} c_{1 x} c_{2 x} g_{y}+\hat{\sigma}_{x} c_{1 y} c_{2 y} g_{x}\right)
$$

$$
\begin{aligned}
& +\frac{(1+\alpha)^{2}}{4}(\hat{\boldsymbol{\sigma}} \cdot \mathbf{g})^{2}\left[\hat{\sigma}_{x} \hat{\sigma}_{y} g_{x} g_{y}-\hat{\sigma}_{x}^{2} c_{1 y} c_{2 y}-\hat{\sigma}_{y}^{2} c_{1 x} c_{2 x}\right] \\
& -\frac{(1+\alpha)^{3}}{8}(\hat{\boldsymbol{\sigma}} \cdot \mathbf{g})^{3}\left(\hat{\sigma}_{x} \hat{\sigma}_{y}^{2} g_{x}+\hat{\sigma}_{x}^{2} \hat{\sigma}_{y} g_{y}\right)-\frac{(1+\alpha)^{4}}{16}(\hat{\boldsymbol{\sigma}} \cdot \mathbf{g})^{4} \hat{\sigma}_{x}^{2} \hat{\sigma}_{y}^{2},
\end{aligned}
$$

$$
\begin{aligned}
{\left[b_{\boldsymbol{\sigma}}(1,2)-1\right] } & c_{1 x} c_{1 y} c_{2 y}^{2}=\frac{1+\alpha}{2}(\hat{\boldsymbol{\sigma}} \cdot \mathbf{g})\left[\hat{\sigma}_{y}\left(2 c_{1 x} c_{1 y} c_{2 y}-c_{1 x} c_{2 y}^{2}\right)-\hat{\sigma}_{x} c_{1 y} c_{2 y}^{2}\right] \\
& +\frac{(1+\alpha)^{2}}{4}(\hat{\boldsymbol{\sigma}} \cdot \mathbf{g})^{2}\left[\hat{\sigma}_{y}^{2}\left(c_{1 x} c_{1 y}-2 c_{1 x} c_{2 y}\right)+\hat{\sigma}_{x} \hat{\sigma}_{y}\left(c_{2 y}^{2}-2 c_{1 y} c_{2 y}\right)\right] \\
& +\frac{(1+\alpha)^{3}}{8}(\hat{\boldsymbol{\sigma}} \cdot \mathbf{g})^{3}\left[\hat{\sigma}_{x} \hat{\sigma}_{y}^{2}\left(2 c_{2 y}-c_{1 y}\right)-\hat{\sigma}_{y}^{3} c_{1 x}\right]+\frac{(1+\alpha)^{4}}{16}(\hat{\boldsymbol{\sigma}} \cdot \mathbf{g})^{4} \hat{\sigma}_{x} \hat{\sigma}_{y}^{3}
\end{aligned}
$$

and

$$
\begin{aligned}
{\left[b_{\boldsymbol{\sigma}}(1,2)-1\right] c_{1 y}^{2} c_{2 y}^{2}=(1+\alpha)(\hat{\boldsymbol{\sigma}} \cdot \mathbf{g}) \hat{\sigma}_{y}\left(c_{1 y}^{2} c_{2 y}-c_{1 y} c_{2 y}^{2}\right) } \\
+\frac{(1+\alpha)^{2}}{4}(\hat{\boldsymbol{\sigma}} \cdot \mathbf{g})^{2} \hat{\sigma}_{y}^{2}\left(c_{1 y}^{2}+c_{2 y}^{2}-4 c_{1 y} c_{2 y}\right) \\
-\frac{(1+\alpha)^{3}}{4}(\hat{\boldsymbol{\sigma}} \cdot \mathbf{g})^{3} \hat{\sigma}_{y}^{3} g_{y}+\frac{(1+\alpha)^{4}}{16}(\hat{\boldsymbol{\sigma}} \cdot \mathbf{g})^{4} \hat{\sigma}_{y}^{4}
\end{aligned}
$$

After multiplying by $\hat{\boldsymbol{\sigma}} \cdot \mathbf{g}$, the $\hat{\boldsymbol{\sigma}}$-integrals can be calculated with the aid of

$$
\begin{aligned}
& \int \mathrm{d} \hat{\boldsymbol{\sigma}} \Theta(\hat{\boldsymbol{\sigma}} \cdot \mathbf{g})(\hat{\boldsymbol{\sigma}} \cdot \mathbf{g})^{2} \hat{\sigma}_{i}=\frac{\pi^{\frac{d-1}{2}}}{\Gamma\left(\frac{d+3}{2}\right)} g g_{i}, \\
& \int \mathrm{d} \hat{\boldsymbol{\sigma}} \Theta(\hat{\boldsymbol{\sigma}} \cdot \mathbf{g})(\hat{\boldsymbol{\sigma}} \cdot \mathbf{g})^{3}=\frac{\pi^{\frac{d-1}{2}}}{\Gamma\left(\frac{d+3}{2}\right)} g^{3}, \\
& \int \mathrm{d} \hat{\boldsymbol{\sigma}} \Theta(\hat{\boldsymbol{\sigma}} \cdot \mathbf{g})(\hat{\boldsymbol{\sigma}} \cdot \mathbf{g})^{3} \hat{\sigma}_{i} \hat{\sigma}_{j}=\frac{\pi^{\frac{d-1}{2}}}{2 \Gamma\left(\frac{d+5}{2}\right)}\left(3 g g_{i} g_{j}+g^{3} \delta_{i j}\right),
\end{aligned}
$$




$$
\begin{aligned}
& \int \mathrm{d} \hat{\boldsymbol{\sigma}} \Theta(\hat{\boldsymbol{\sigma}} \cdot \mathbf{g})(\hat{\boldsymbol{\sigma}} \cdot \mathbf{g})^{4} \hat{\sigma}_{i}=\frac{2 \pi^{\frac{d-1}{2}}}{\Gamma\left(\frac{d+5}{2}\right)} g^{3} g_{i}, \\
& \int \mathrm{d} \hat{\boldsymbol{\sigma}} \Theta(\hat{\boldsymbol{\sigma}} \cdot \mathbf{g})(\hat{\boldsymbol{\sigma}} \cdot \mathbf{g})^{4} \hat{\sigma}_{y}^{2} \hat{\sigma}_{j}=\frac{\pi^{\frac{d-1}{2}}}{\Gamma\left(\frac{d+7}{2}\right)}\left[3 g g_{y}^{2} g_{j}+g^{3} g_{j}+2 g^{3} g_{y} \delta_{y j}\right], \\
& \int \mathrm{d} \hat{\boldsymbol{\sigma}} \Theta(\hat{\boldsymbol{\sigma}} \cdot \mathbf{g})(\hat{\boldsymbol{\sigma}} \cdot \mathbf{g})^{4} \hat{\sigma}_{x} \hat{\sigma}_{y} \hat{\sigma}_{z}=\frac{3 \pi^{\frac{d-1}{2}}}{\Gamma\left(\frac{d+7}{2}\right)} g g_{x} g_{y} g_{z}, \\
& \int \mathrm{d} \hat{\boldsymbol{\sigma}} \Theta(\hat{\boldsymbol{\sigma}} \cdot \mathbf{g})(\hat{\boldsymbol{\sigma}} \cdot \mathbf{g})^{5} \hat{\sigma}_{i} \hat{\sigma}_{j}=\frac{\pi^{\frac{d-1}{2}}}{\Gamma\left(\frac{d+7}{2}\right)}\left(5 g^{3} g_{i} g_{j}+g^{5} \delta_{i j}\right),
\end{aligned}
$$

$\int \mathrm{d} \hat{\boldsymbol{\sigma}} \Theta(\hat{\boldsymbol{\sigma}} \cdot \mathbf{g})(\hat{\boldsymbol{\sigma}} \cdot \mathbf{g})^{5} \hat{\sigma}_{y}^{3} \hat{\sigma}_{j}=\frac{3 \pi^{\frac{d-1}{2}}}{2 \Gamma\left(\frac{d+9}{2}\right)}\left[5 g g_{y}\left(g^{2}+g_{y}^{2}\right) g_{j}+g^{3}\left(g^{2}+5 g_{y}^{2}\right) \delta_{y j}\right]$,

calculated for arbitrary dimension and

$$
\int \mathrm{d} \hat{\boldsymbol{\sigma}} \Theta(\hat{\boldsymbol{\sigma}} \cdot \mathbf{g})(\hat{\boldsymbol{\sigma}} \cdot \mathbf{g})^{5} \hat{\sigma}_{x}^{2} \hat{\sigma}_{j}^{2}=\frac{3 \pi^{\frac{1}{2}}}{2 \Gamma\left(\frac{11}{2}\right)}\left[g^{3}\left(g^{2}+g_{x}^{2}\right)+8 g^{3} g_{x} g_{j} \delta_{x j}+\left(g^{3}+5 g g_{x}^{2}\right) g_{j}^{2}\right],
$$

for $d=2$.

In the following we will restrict ourselves to the case $d=2$ and will use the Jenkins and Richman distribution to $\epsilon^{2}$ order [20]

$$
\chi(\mathbf{c}) \approx \frac{\mathrm{e}^{-c^{2}}}{\pi}\left[1-\epsilon \sqrt{2} c_{x} c_{y}+\epsilon^{2}\left(\frac{1}{4}-c_{y}^{2}+c_{x}^{2} c_{y}^{2}\right)\right] .
$$

To the same approximation, we have

$$
\begin{aligned}
\chi\left(\mathbf{c}_{1}\right) \chi\left(\mathbf{c}_{2}\right) \approx & \frac{1}{\pi^{2}} \mathrm{e}^{-c_{1}^{2}-c_{2}^{2}}\left[1-\epsilon \sqrt{2}\left(c_{1 x} c_{1 y}+c_{2 x} c_{2 y}\right)\right. \\
& \left.+\epsilon^{2}\left(\frac{1}{4}-c_{1 y}^{2}-c_{2 y}^{2}+c_{1 x}^{2} c_{1 y}^{2}+c_{2 x}^{2} c_{2 y}^{2}+2 c_{1 x} c_{1 y} c_{2 x} c_{2 y}\right)\right],
\end{aligned}
$$

or, in terms of the new variables $\{\mathbf{g}, \mathbf{G}\}$

$$
\begin{aligned}
& \chi\left(\mathbf{c}_{1}\right) \chi\left(\mathbf{c}_{2}\right) \approx \frac{\mathrm{e}^{-\frac{1}{2} g^{2}-2 G^{2}}}{\pi^{2}}\left\{1-\frac{\epsilon}{\sqrt{2}}\left(g_{x} g_{y}+4 G_{x} G_{y}\right)\right. \\
& \left.+\frac{\epsilon^{2}}{4}\left[2+\left(g_{x}^{2}-2\right) g_{y}^{2}+8 g_{x} g_{y} G_{x} G_{y}+8\left(2 G_{x}^{2}-1\right) G_{y}^{2}\right]\right\} .
\end{aligned}
$$

The velocity integrals given by equation (C.8) can be calculated with the aid of Mathematica, obtaining, up to $\epsilon^{2}$

$$
\begin{aligned}
& T_{22}=-\frac{3}{2} \sqrt{\frac{\pi}{2}} \epsilon^{2}, \\
& T_{23}=\frac{5}{8} \sqrt{\pi} \epsilon, \\
& T_{24}=-\frac{1}{8} \sqrt{\frac{\pi}{2}} \epsilon^{2},
\end{aligned}
$$


Fluctuations in the uniform shear flow state of a granular gas

$$
\begin{aligned}
T_{33} & =-\frac{19}{64} \sqrt{\frac{\pi}{2}} \epsilon^{2}, \\
T_{34} & =\frac{5}{16} \sqrt{\pi} \epsilon, \\
T_{44} & =\frac{11}{64} \sqrt{\frac{\pi}{2}} \epsilon^{2} .
\end{aligned}
$$

Finally, by substituting the expressions for the $T_{i j}$ coefficients obtained above into equation (C.7) and that into equations (C.1)-(C.6), we obtain the linear system for $C_{i j}$ mentioned above. This system is solved with the aid of Mathematica to obtain the expressions in the main text.

\section{References}

[1] Dufty J W 2000 J. Phys.: Condens. Matter 12 A47

[2] Goldhirsch I 2003 Annu. Rev. Fluid Mech. 35267

[3] Brilliantov N and Poeschel T 2004 Kinetic Theory of Granular Gases (Oxford: Clarendon)

[4] Lutsko J F 2005 Phys. Rev. E 72021306

[5] Aranson I S and Tsimring L S 2006 Rev. Mod. Phys. 78641

[6] Résibois P and de Leener M 1977 Classical Kinetic Theory of Fluids (New York: Wiley)

[7] Goldshtein A and Shapiro M 1995 J. Fluid. Mech. 28275

[8] Brey J J, Dufty J W and Santos A 1997 J. Stat. Phys. 871051

[9] Brey J J, García de Soria M I, Maynar P and Ruiz-Montero M J 2004 Phys. Rev. E 70011302

[10] Brey J J, Dufty J W, Kim C S and Santos A 1998 Phys. Rev. E 584638

Brey J J and Cubero D 2001 Granular Gases (Lecture Notes in Physics vol 564) ed T Poeschel et al (Berlin: Springer)

[11] Brey J J, Dufty J W and Ruiz-Montero M J 2003 Granular Gas Dynamics (Lecture Notes in Physics vol 624) ed T Poeschel et al (Berlin: Springer)

[12] Dufty J W and Brey J J 2003 Phys. Rev. E 68030302

[13] Brey J J and Dufty J W 2005 Phys. Rev. E 72011303

[14] Haff P K 1983 J. Fluid. Mech. 134401

[15] Brey J J, Maynar P and García de Soria M I 2009 Phys. Rev. E 79051305

[16] Brey J J, Maynar P and García de Soria M I 2011 Phys. Rev. E 83041303

[17] Kubo R, Toda M and Hashitsume N 1987 Statistical Physics II (Berlin: Springer)

[18] Brey J J, García M I de Soria and Maynar P 2012 Phys. Rev. E 86031304

[19] Lun C K K, Savage S B, Jeffrey D J and Chepurniy N 1984 J. Fluid Mech. 140223

[20] Jenkins J T and Richman M W 1988 J. Fluid Mech. 192313

[21] Sela N, Goldhirsch I and Noskowicz S H 1996 Phys. Fluids 82337

[22] Brey J J, Ruiz-Montero M J and Moreno F 1997 Phys. Rev. E 552846

[23] Garzó V and Santos A 2003 Kinetic Theory of Gases in Shear Flows. Nonlinear Transport (Dordrecht: Kluwer)

[24] Santos A, Garzó V and Dufty J W 2004 Phys. Rev. E 69061303

[25] Ernst M H and Cohen E G D 1981 J. Stat. Phys. 25153

[26] Dufty J W and Brey J J 2006 Modelling and Numerics of Kinetic Dissipative Systems ed L Pareschi et al (New York: Nova Science)

[27] Lees A W and Edwards S F 1972 J. Phys. C 51921

[28] Dufty J W, Santos A, Brey J J and Rodríguez R F 1986 Phys. Rev. A 33459

[29] García de Soria M I, Maynar P and Trizac E 2009 Mol. Phys. 107383

[30] Maynar P, García de Soria M I, Schehr G, Barrat A and Trizac E 2008 Phys. Rev. E 77051128

[31] Astillero A and Santos A 2007 Europhys. Lett. 781

[32] Astillero A and Santos A 2012 Phys. Rev. E 85021302

[33] Brey J J, Maynar P and García de Soria M I 2012 Phys. Rev. E 86061308

[34] Garzó V 2006 Phys. Rev. E 73021304

[35] Brey J J, García de Soria M I and Maynar P 2010 Phys. Rev. E 82021303

[36] García de Soria M I and Maynar P to be published 